Preprint typeset in JHEP style - HYPER VERSION

ITFA-2007-28

\title{
Classical approximation to quantum cosmological correlations
}

\author{
Meindert van der Meulen and Jan Smit \\ Institute for Theoretical Physics, University of Amsterdam, \\ Valckenierstraat 65, 1018 XE Amsterdam, The Netherlands.
}

\begin{abstract}
We investigate up to which order quantum effects can be neglected in calculating cosmological correlation functions after horizon exit. As a toy model, we study $\phi^{3}$ theory on a de Sitter background for a massless minimally coupled scalar field $\phi$. We find that for tree level and one loop contributions in the quantum theory, a good classical approximation can be constructed, but for higher loop corrections this is in general not expected to be possible. The reason is that loop corrections get non-negligible contributions from loop momenta with magnitude up to the Hubble scale $H$, at which scale classical physics is not expected to be a good approximation to the quantum theory. An explicit calculation of the one loop correction to the two point function, supports the argument that contributions from loop momenta of scale $H$ are not negligible. Generalization of the arguments for the toy model to derivative interactions and the curvature perturbation leads to the conclusion that the leading orders of non-Gaussian effects generated after horizon exit, can be approximated quite well by classical methods. Furthermore we compare with a theorem by Weinberg. We find that growing loop corrections after horizon exit are not excluded, even in single field inflation.
\end{abstract}

KEYWORDS: quantum field theory on curved space, inflation, physics of the early universe, CMBR theory. 


\section{Contents}

1. Introduction 2

2. Quantum theory 1

2.1 Closed Time Path formalism on a de Sitter background

2.1.1 CTP formalism

2.1.2 Feynman rules on a de Sitter background 6

2.1.3 Example: equal time two point function

2.2 Late times 8

2.2.1 Cosmological correlation functions 8

2.2.2 Small internal momenta 9

2.2.3 Large internal momenta 10

2.2.4 Example: Late time contributions to equal time two point function at one loop $\quad 12$

3. Classical theory 13

3.1 Perturbative calculation of correlation functions 13

3.2 Graphical representation 14

4. Classical approximation 16

4.1 Small internal momenta 16

4.2 Large internal momenta 17

4.3 Classical approximation at one loop 17

4.4 Example 18

5. Discussion and conclusions 19

5.1 Early time contributions 19

5.2 Generalization to derivative interactions 19

5.3 Comparison to stochastic approach 20

5.3.1 Massless minimally coupled scalar with nonderivative interactions 21

5.3.2 Derivative interactions and other fields 23

5.4 Application to the curvature perturbation 23

5.5 Conclusions 24

A. Free scalar field on a de Sitter background 25

A.1 Scalar wavefunction 25

A.2 Particle creation 26

A.3 Comparison with thermal field theory 27

B. Amputated diagrams with no external dashed lines 28 
D. One loop correction to two point function

D.1 Diagrams A and D 30

D.1.1 Amputated diagram for small internal momenta 30

D.1.2 Amputated diagrams for large internal momenta 31

D.1.3 Attaching the external lines 36

D.2 Diagrams B and C 37

D.2.1 Amputated diagrams for small internal momenta 37

D.2.2 Amputated diagrams for large internal momenta 38

D.2.3 Attaching the external lines 40

\section{Introduction}

The precision of measurements of temperature fluctuations in the Cosmic Microwave Background radiation has increased enormously in the recent past and is expected to increase even more in the near future. From these measurements, statistical properties of the primordial cosmological perturbations can be deduced. They are found to have a nearly flat power spectrum, and to be close to Gaussian. Non-Gaussian effects (see [1] for a review) might be detected in the future and can provide a powerful tool to discriminate between different inflation models.

There is therefore a large interest in calculating the statistical properties of the primordial cosmological perturbations for different inflation models. In these calculations the cosmological perturbations are often parameterized by the curvature perturbation $\zeta$, which is the perturbation in scalar curvature on time slices of uniform density. This parameterization is convenient because of the property that, under certain conditions, perturbations in $\zeta$ remain constant after their wavelengths have grown larger than the horizon length, i.e. after horizon exit. This has been shown for linear perturbation theory in [2], to all orders in $\zeta$ for single field inflation in [3], and nonperturbatively for adiabatic perturbations in [4]. The latter two references use a derivative expansion and therefore assume that effects from wavelengths of the order of the horizon and shorter, are negligible.

In more complicated models for inflation, e.g. those involving multiple fields, the curvature perturbation $\zeta$ is not constant after horizon exit. Therefore evolution after horizon exit might lead to non-Gaussian effects, which has been investigated in [5, 6, 7, 8, 9, 10, 11, 12, 13, 14, 15, 16, 17, 18, 19, 20, 21, 22, 23, 24, 25, 26, 27. These investigations have been done by solving classical equations of motion, which is assumed to be a good approximation to the quantum theory, because quantum effects are presumably negligible for wavelengths much longer than the horizon length (see [28] for a recent argument).

The goal of this paper is to investigate up to which order corrections to cosmological correlation functions that are generated after horizon exit, can be calculated reliably using classical physics. For this we study $\phi^{3}$ theory on an exact de Sitter background for a 
massless minimally coupled scalar field $\phi$, as toy model for the curvature perturbation $\zeta$ on an inflationary background. We consider correlation functions with (external) momenta much smaller than the Hubble scale $H$ in the quantum theory, using the Closed Time Path (CTP) formalism (also known as in-in formalism, see e.g. [29, 30]), as is also done by Weinberg in [31, 32]. Furthermore we formulate a classical theory with statistical fluctuations, in such a way that correlation functions in this theory can easily be compared with those in the quantum theory.

In short our results for the $\phi^{3}$ toy model are that the tree level contributions in the quantum theory can be approximated quite well by classical physics (which sounds trivial, but we argue that this is not completely so). However we find that the classical approximation is not expected to be good in general for loop corrections in the quantum theory. The reason is that loop integrals get contributions from momenta with magnitude up to the Hubble scale, and the contributions from loop momenta at scales around $H$ are in general not negligible. This is supported by an explicit calculation of the one loop correction to the two point function, where we use a small mass as infrared regulator. We argue that the classical approximation is not expected to be good at scales around $H$, and can therefore in general not reproduce these contributions. An exception is the one loop correction, for which we find that the classical approximation can be saved by choosing a suitable ultraviolet cutoff.

We generalize the arguments for the toy model to derivative interactions, and apply them to the curvature perturbation $\zeta$. This leads to the conclusion that non-Gaussian effects generated after horizon exit in multifield inflation models can, up to one loop level, be approximated by classical physics. We also compare with a theorem derived by Weinberg [31]. We find that it is not excluded that there are corrections to correlation functions of $\zeta$ that grow after horizon exit, even in single field inflation.

We remark that the problem we are addressing, is related to, but different from the problem of the quantum-to-classical transition [33, 34, 35, 36, 37, 38], that deals with the way how quantum fluctuations acquire classical properties by decoherence, and with the production of entropy. In this paper we are not investigating how a quantum system evolves to a classical system; we are considering a quantum system and a classical system separately from each other and investigate how well the classical system can reproduce correlation functions of the quantum system.

In the next section we recall the CTP formalism, using a variation of the Keldyshbasis, and apply it to $\phi^{3}$ theory on a de Sitter background (for other applications of the CTP formalism to interacting fields in cosmology see [39, 40, 41, 42, 43, 44, 45, 46, 47, 48, 49, 50, 51, 52, 53, 54, 55, 56]). Subsequently we analyze contributions to correlation functions with small external momenta, that are generated after horizon exit (but still during inflation). In section 3 we show how correlation functions in classical $\phi^{3}$ theory on a de Sitter background can be calculated perturbatively, starting from given initial conditions. The perturbative contributions are graphically represented in a way that is similar to the Feynman diagrams of the quantum theory. Next in section we argue that the classical theory can approximate the contributions from small internal momenta in the quantum theory quite well, but that this is in general not the case for large internal 
momenta. The one loop correction is an exception: we show that by choosing a suitable ultraviolet cutoff, the classical approximation can be good. We generalize our arguments and conclude in section 5 .

In Appendix A details of the quantization are given and a comparison is made with finite temperature field theory. Appendix B contains an argument on closed retarded loops. In Appendix 9 diagrams in the classical and quantum theory are compared. To illustrate the arguments in this paper, we give in Appendix D the detailed calculations of the one loop correction to the two point function in the $\phi^{3}$ toy model, that is generated after horizon exit.

\section{Quantum theory}

In this section we set up the quantum theory using the CTP formalism, and analyze the contributions to correlation functions that are generated after horizon exit.

The Lagrangian density of $\phi^{3}$ theory is

$$
\mathcal{L}[\phi]=\sqrt{-g}\left(-\frac{1}{2} \partial_{\mu} \phi \partial^{\mu} \phi-\frac{1}{2} m^{2} \phi^{2}-\frac{1}{2} \xi R \phi^{2}-\frac{\lambda}{3 !} \phi^{3}\right)+\delta \mathcal{L}
$$

where we are using a metric $g_{\mu \nu}$ with signature -+++ . Except when we need the mass $m$ as infrared regulator, we take $m=0$ and $\xi=0$ to obtain a massless minimally coupled scalar field. The term $\delta \mathcal{L}$ contains the counterterms:

$$
\delta \mathcal{L}=\sqrt{-g}\left(-\delta_{1} \phi-\frac{1}{2} \delta_{Z} \partial_{\mu} \phi \partial^{\mu} \phi-\frac{1}{2} \delta_{m} \phi^{2}-\frac{\delta_{\lambda}}{3 !} \phi^{3}\right)
$$

We added a linear counterterm $\delta_{1}$ to keep $\langle\phi\rangle=0$ for all times, at one loop level ${ }^{1}$, hence up to order $\mathcal{O}\left(\lambda^{3}\right)$. The potential can be stabilized by adding a $\phi^{4}$ term if desired. We use a spatial momentum cutoff $\Lambda$ as ultraviolet regulator. Then

$$
\delta_{m}=\frac{\lambda^{2}}{4(2 \pi)^{2}} \ln \frac{\Lambda}{\mu}+\mathcal{O}\left(\lambda^{4}\right)
$$

where $\mu$ is a renormalization scale.

\subsection{Closed Time Path formalism on a de Sitter background}

\subsubsection{CTP formalism}

In a system with time-dependent Hamiltonian $H(t)$, that starts in a state |in $\rangle$ at initial time $t_{i}$, the expectation value of an operator $Q$ at time $t>t_{i}$ is given by

$$
\langle Q(t)\rangle=\left\langle\operatorname{in}\left|\left[\overline{\mathrm{T}} \exp \left(i \int_{t_{\mathrm{in}}}^{t} d t^{\prime} H\left(t^{\prime}\right)\right)\right] Q\left[\mathrm{~T} \exp \left(-i \int_{t_{\mathrm{in}}}^{t} d t^{\prime} H\left(t^{\prime}\right)\right)\right]\right| \text { in }\right\rangle,
$$

\footnotetext{
${ }^{1}$ It can be checked that this is possible by calculating the tadpole diagram, using the $F$ two point function of equation (2.24) and the infrared and ultraviolet regulators as discussed later in this paper.
} 
where $\mathrm{T}$ means a time-ordered product and $\overline{\mathrm{T}}$ an anti-time-ordered product. In the Closed Time Path (CTP) formalism (or in-in formalism) [30] this expectation value can also be calculated using path integrals, from the generating functional

$$
\begin{aligned}
& Z\left[J_{+}, J_{-}, \rho\left(t_{\text {in }}\right)\right]=\int \mathcal{D} \phi_{\text {in }}^{+} \mathcal{D} \phi_{\text {in }}^{-}\left\langle\phi_{\text {in }}^{+}\left|\rho\left(t_{\text {in }}\right)\right| \phi_{\text {in }}^{-}\right\rangle \times \\
& \int_{\phi_{\text {in }}^{+}}^{\phi_{\text {in }}^{-}} \mathcal{D} \phi^{+} \mathcal{D} \phi^{-} \exp \left[i \int_{t_{\text {in }}}^{t} d t^{\prime} \int d^{3} x\left(\mathcal{L}\left[\phi^{+}\right]-\mathcal{L}\left[\phi^{-}\right]+J_{+} \phi^{+}+J_{-} \phi^{-}\right)\right] .
\end{aligned}
$$

The path integral on the second line can be written in short-hand notation as

$$
\int \mathcal{D} \phi \exp \left[i \int_{\mathcal{C}} d t^{\prime} \int d^{3} x(\mathcal{L}[\phi]+J \phi)\right]
$$

where $\mathcal{C}$ is the so-called Schwinger-Keldysh contour which runs from $t_{\text {in }}$ to $t$ and back. The field $\phi$ and source $J$ are split up in $\phi^{+}, J_{+}$on the first part of this contour, and $\phi^{-}, J_{-}$ on the second part, with the condition $\phi^{+}(t)=\phi^{-}(t)$. The integration along the contour $\mathcal{C}$ explains the name Closed Time Path formalism. The path integral on the first line of equation (2.5) imposes that at the initial time $t_{\text {in }}$, the state of the system is given by the density matrix $\rho\left(t_{\text {in }}\right)$. Expectation values are then obtained by variation of the sources $J_{+}$ and $J_{-}$:

$$
\begin{aligned}
& \left\langle\overline{\mathrm{T}}\left(\phi\left(x_{1}\right) \ldots \phi\left(x_{n}\right)\right) \mathrm{T}\left(\phi\left(x_{n+1}\right) \ldots \phi\left(x_{n+m}\right)\right)\right\rangle= \\
& \left.\frac{\delta^{n+m} Z\left[J_{+}, J_{-}, \rho\left(t_{\text {in }}\right)\right]}{\delta J_{-}\left(x_{1}\right) \cdots \delta J_{-}\left(x_{n}\right) \delta J_{+}\left(x_{n+1}\right) \cdots \delta J_{+}\left(x_{n+m}\right)}\right|_{J_{+}, J_{-}=0},
\end{aligned}
$$

where the times $x_{j}^{0}$ are smaller than or equal to the time $t$ used in definition (2.5).

When calculating these correlation functions perturbatively, we need to know the free two point functions with all four possible time orderings:

$$
\begin{aligned}
& G^{-+}(x, y)=i\langle\phi(x) \phi(y)\rangle^{(0)}, \\
& G^{+-}(x, y)=i\langle\phi(y) \phi(x)\rangle^{(0)}, \\
& G^{++}(x, y)=i\langle\operatorname{T} \phi(x) \phi(y)\rangle^{(0)}=\theta\left(x_{0}-y_{0}\right) G^{-+}(x, y)+\theta\left(y_{0}-x_{0}\right) G^{+-}(x, y), \\
& G^{--}(x, y)=i\langle\overline{\mathrm{T}} \phi(x) \phi(y)\rangle^{(0)}=\theta\left(x_{0}-y_{0}\right) G^{+-}(x, y)+\theta\left(y_{0}-x_{0}\right) G^{-+}(x, y),
\end{aligned}
$$

where the superscript (0) denotes the free field correlation functions. They obey the identity

$$
G^{++}(x, y)+G^{--}(x, y)=G^{-+}(x, y)+G^{+-}(x, y)
$$

and they can be put together in a matrix:

$$
\mathbf{G}(x, y)=\left(\begin{array}{ll}
G^{++}(x, y) & G^{+-}(x, y) \\
G^{-+}(x, y) & G^{--}(x, y)
\end{array}\right) .
$$

Note that the two point functions depend on the initial conditions via the dependence on $\rho\left(t_{i}\right)$ of the generating functional (2.5). 
In the context of the classical approximation it is useful to transform the $\phi^{+}$and $\phi^{-}$ fields to a different basis, which is a variation of the Keldysh basis (see also [57]):

$$
\left(\begin{array}{c}
\phi^{(1)} \\
\phi^{(2)}
\end{array}\right)=\left(\begin{array}{c}
\left(\phi^{+}+\phi^{-}\right) / 2 \\
\phi^{+}-\phi^{-}
\end{array}\right)=\mathbf{R}\left(\begin{array}{c}
\phi^{+} \\
\phi^{-}
\end{array}\right), \quad \text { with } \quad \mathbf{R}=\left(\begin{array}{cc}
1 / 2 & 1 / 2 \\
1 & -1
\end{array}\right) .
$$

The Lagrangian density $\mathcal{L}\left[\phi^{+}\right]-\mathcal{L}\left[\phi^{-}\right]$transforms to

$\mathcal{L}\left[\phi^{(1)}, \phi^{(2)}\right]=\sqrt{-g}\left(-\partial_{\mu} \phi^{(1)} \partial^{\mu} \phi^{(2)}-\left(m^{2}+\xi R\right) \phi^{(1)} \phi^{(2)}-\frac{\lambda}{3 !}\left(3\left(\phi^{(1)}\right)^{2} \phi^{(2)}+\frac{1}{4}\left(\phi^{(2)}\right)^{3}\right)\right)$.

The free two point functions in this basis can easily be obtained by the transformation

$$
\mathbf{G}_{K}(x, y)=\mathbf{R G}(x, y) \mathbf{R}^{T}=\left(\begin{array}{cc}
i F(x, y) & G^{R}(x, y) \\
G^{A}(x, y) & 0
\end{array}\right),
$$

with

$$
\begin{aligned}
F(x, y) & =-\frac{i}{2}\left(G^{-+}(x, y)+G^{+-}(x, y)\right) \\
G^{R}(x, y) & =G^{++}(x, y)-G^{+-}(x, y)=\theta\left(x_{0}-y_{0}\right)\left(G^{-+}(x, y)-G^{+-}(x, y)\right) \\
G^{A}(x, y) & =G^{++}(x, y)-G^{-+}(x, y)=\theta\left(y_{0}-x_{0}\right)\left(G^{+-}(x, y)-G^{-+}(x, y)\right)
\end{aligned}
$$

where we have used identity (2.12). They obey the equations

$$
\begin{aligned}
\left(\square_{x}+m^{2}+\xi R(x)\right) F(x, y) & =0 \\
\left(\square_{x}+m^{2}+\xi R(x)\right) G^{R, A}(x, y) & =\frac{\delta^{4}(x-y)}{\sqrt{-g(x)}},
\end{aligned}
$$

with

$$
\square_{x}=\frac{1}{\sqrt{-g(x)}} \partial_{\mu}\left(\sqrt{-g(x)} g^{\mu \nu}(x) \partial_{\nu}\right) .
$$

The $G^{R}$ and $G^{A}$ two point functions are often called the retarded and advanced propagators. Note that $G^{A}(x, y)=G^{R}(y, x)$.

\subsubsection{Feynman rules on a de Sitter background}

The metric of the de Sitter background is

$$
d s^{2}=-d t^{2}+a^{2}(t) d \mathbf{x}^{2}
$$

where $a(t)$ is the FRW scale factor. The Hubble rate is $H=\dot{a} / a$. In de Sitter space the scale factor is $a(t)=a_{0} \exp (H t)$. We will use conformal time $\tau=-\int_{t}^{\infty} d t^{\prime} / a\left(t^{\prime}\right)$, which runs from $-\infty$ to 0 . The scale factor in conformal time is $a(\tau)=-1 / H \tau$.

As initial state $\rho\left(\tau_{\text {in }}\right)$ we take the adiabatic or Bunch-Davies vacuum, for $\tau_{\text {in }} \rightarrow-\infty$. One expects that other choices will give the same results because this state is an attractor 
state [58, 59]. The free field operator with this initial state is given in equation (A.5) in Appendix $\mathrm{A}$ and it can be used to derive the free two point functions (2.17)-(2.19):

$$
\begin{aligned}
F\left(k, \tau_{1}, \tau_{2}\right) & =\frac{H^{2}}{2 k^{3}}\left[\left(1+k^{2} \tau_{1} \tau_{2}\right) \cos k\left(\tau_{1}-\tau_{2}\right)+k\left(\tau_{1}-\tau_{2}\right) \sin k\left(\tau_{1}-\tau_{2}\right)\right], \\
G^{R}\left(k, \tau_{1}, \tau_{2}\right) & =\theta\left(\tau_{1}-\tau_{2}\right) \frac{H^{2}}{k^{3}}\left[\left(1+k^{2} \tau_{1} \tau_{2}\right) \sin k\left(\tau_{1}-\tau_{2}\right)-k\left(\tau_{1}-\tau_{2}\right) \cos k\left(\tau_{1}-\tau_{2}\right)\right],
\end{aligned}
$$

and $G^{A}\left(k, \tau_{1}, \tau_{2}\right)=G^{R}\left(k, \tau_{2}, \tau_{1}\right)$, and where the two point functions depend only on the length of the spatial momentum $k=|\mathbf{k}|$. Representing the $\phi^{(1)}$ field with a full line and the $\phi^{(2)}$ field with a dashed line, the Feynman rules for the two point functions, the vertices and the counterterm are ${ }^{2}$

$$
\begin{aligned}
& \frac{\tau_{1} \tau_{2}}{\tau_{1}}=F\left(k, \tau_{1}, \tau_{2}\right), \\
& \frac{\tau_{2}}{\tau_{1}}=-i G^{R}\left(k, \tau_{1}, \tau_{2}\right)=-i G^{A}\left(k, \tau_{2}, \tau_{1}\right), \\
& \tau_{\tau_{2}}^{\tau_{2}}=-i \lambda a^{4}\left(\tau_{1}\right) \delta\left(\tau_{1}-\tau_{2}\right) \delta\left(\tau_{1}-\tau_{3}\right), \\
& \tau_{\tau_{3},}^{\tau_{2}}=-\frac{i \lambda}{4} a^{4}\left(\tau_{1}\right) \delta\left(\tau_{1}-\tau_{2}\right) \delta\left(\tau_{1}-\tau_{3}\right), \\
& \tau_{-}^{\tau_{2}}=-i a^{4}\left(\tau_{1}\right) \delta\left(\tau_{1}-\tau_{2}\right) \delta_{m} .
\end{aligned}
$$

When a two point function is attached to a vertex, the corresponding time has to be integrated over. A closed loop corresponds with an integral over spatial momentum $\int d^{3} p /(2 \pi)^{3}$.

\subsubsection{Example: equal time two point function}

As an example to which we will return repeatedly, we consider the equal time two point function up to one loop level:

$$
\int d^{3} x e^{-i \mathbf{k} \cdot \mathbf{x}}\langle\phi(\tau, \mathbf{x}) \phi(\tau, \mathbf{0})\rangle
$$

The tree level contribution is given by

$$
\tau \quad \tau(k, \tau, \tau) .
$$

There is no contribution with the $G^{R}$ two point function because that vanishes for equal times. At one loop level there are contributions

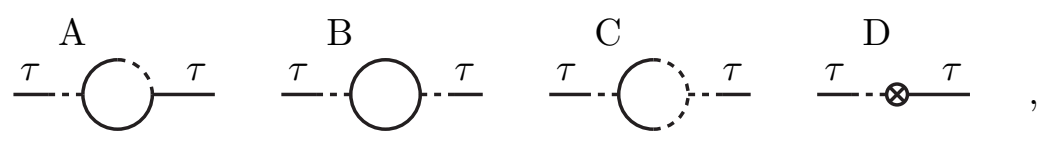

\footnotetext{
${ }^{2}$ These Feynman rules should not be confused with the graphical representation developed in 60 .
} 
where diagrams $\mathrm{A}, \mathrm{C}$ and $\mathrm{D}$ have also mirror versions which correspond to interchanging the endpoints. There is also the diagram

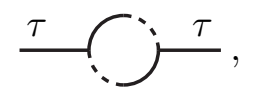

but it vanishes because of the $\theta$-functions in the $G^{R}$ two point functions. This is an example of the general fact that diagrams with no external $G^{R}$ two point functions vanish, as explained in Appendix B. Furthermore there are diagrams with dashed lines at the endpoints, but these diagrams vanish also because of $\theta$-functions. Diagrams with tadpoles are canceled by the linear counterterm $\delta_{1}$.

Using the Feynman rules, the diagrams can be translated to integrals. For example diagram A translates to

$$
\begin{array}{r}
\frac{\tau}{-1 j \tau}=(-i)^{2}(-i \lambda)^{2} \int_{\tau_{\text {in }}}^{\tau} d \tau_{1} a^{4}\left(\tau_{1}\right) \int_{\tau_{\text {in }}}^{\tau} d \tau_{2} a^{4}\left(\tau_{2}\right) G^{R}\left(k, \tau, \tau_{1}\right) F\left(k, \tau, \tau_{2}\right) \times \\
\int \frac{d^{3} p}{(2 \pi)^{3}} G^{R}\left(|\mathbf{k}+\mathbf{p}|, \tau_{1}, \tau_{2}\right) F\left(p, \tau_{1}, \tau_{2}\right) .
\end{array}
$$

The symmetry factor is 1 because the propagators in the loop are different (diagrams $\mathrm{B}$ and $\mathrm{C}$ have symmetry factor $1 / 2$ ). The momentum integral is both infrared and ultraviolet divergent. The ultraviolet divergence is the same as the one that occurs in Minkowski space and is canceled by the counterterm in diagram $\mathrm{D}$. The infrared divergence has to be regularized, e.g. by giving $\phi$ a small mass or by taking space to be finite.

In Appendix D the one loop correction (2.33) is calculated explicitly, using an initial time $\tau_{H}$ with $\left|k \tau_{H}\right|<1$.

\subsection{Late times}

\subsubsection{Cosmological correlation functions}

In this paper we consider cosmological correlation functions, by which we mean equal time correlation functions

$$
\int d^{3} x_{1} \ldots d^{3} x_{r} e^{-i \mathbf{k}_{1} \cdot \mathbf{x}_{1}-\ldots-i \mathbf{k}_{r} \cdot \mathbf{x}_{r}}\left\langle\phi\left(\tau, \mathbf{x}_{1}\right) \ldots \phi\left(\tau, \mathbf{x}_{r}\right) \phi(\tau, \mathbf{0})\right\rangle
$$

where the time $\tau$ is late, i.e. is well after horizon exit with respect to the spatial momenta $k_{i}$, which can be expressed as $\left|k_{i} \tau\right| \ll 1$. Similarly, early times are times for which $\left|k_{i} \tau\right| \gg 1$.

We constrain the analysis further by only considering contributions to these cosmological correlation functions, that are generated after horizon exit. For this we introduce a split in time at $\tau_{H}$, a few $\left(N_{H}\right)$ e-folds after horizon exit, such that $\left|k \tau_{H}\right|=\exp \left(-N_{H}\right) \ll 1$. Correlation functions at $\tau_{H}$ have accumulated contributions from earlier times, of which we only keep the free field contributions. Then we use these correlation functions as initial conditions for the evolution after $\tau_{H}$. In practice this means that we use the Feynman rules as described in the previous subsection, with the only difference that we take $\tau_{H}$ as 
initial time. This procedure is not completely correct because the neglected contributions generated before $\tau_{H}$ are of the same order in the coupling constant as the contributions generated after $\tau_{H}$. But it does not change the qualitative behaviour of the contributions generated after $\tau_{H}$, and neglecting the contributions from before $\tau_{H}$ simplifies the calculations significantly. We comment further on this point in section 5.1.

Different contributions to cosmological correlation functions (2.36) depend in different ways on the time $\tau$. Those that are proportional to positive powers of $\tau$, are dominated by their values at the initial time $\tau_{H}$. These contributions are negligible if $\tau_{H}$ is taken sufficiently long after horizon exit. Contributions that are proportional to a non-positive power of $\tau$ can grow after horizon exit and will therefore dominate. In [31, 32] it is shown that these contributions do not grow faster than powers of $\ln (-H \tau)$, so negative powers of $\tau$ do not occur. In this paper we call contributions that are proportional to $\tau^{0}$ (including powers of $\ln (-H \tau))$ late time contributions. In this subsection we analyze the dependence on $\tau$ of the different contributions by counting powers of $\tau$.

Contributions can contain integrals over spatial internal (loop) momenta, $p$, which can be arbitrarily large. We have found that the power counting goes quite differently for small internal momenta (smaller than the Hubble scale $H$, i.e. $|p \tau| \ll 1$ ), than for large internal momenta (of the order of the Hubble scale and larger, i.e. $|p \tau| \gtrsim 1$ ). Therefore we analyze first the case that all internal momenta are small, and consider then arbitrary (amputated) 1PI diagrams for which all the internal momenta are large and the external momenta are small. These 1PI diagrams can be treated as effective (non-local) couplings in the analysis for small internal momenta, and in this way our analysis covers the whole range of internal momenta. After this general analysis, we compare our results with the specific case of the one loop correction to the two point function.

\subsubsection{Small internal momenta}

When the internal momenta are small, the expressions for the free two point functions $F$ and $G^{R}(2.24)$, 2.25) can be expanded in $k \tau_{i}$ :

$$
\begin{aligned}
F\left(k, \tau_{1}, \tau_{2}\right) & =\frac{H^{2}}{2 k^{3}}\left[1+\mathcal{O}\left(k^{2} \tau_{i}^{2}\right)\right], \\
G^{R}\left(k, \tau_{1}, \tau_{2}\right) & =\theta\left(\tau_{1}-\tau_{2}\right) \frac{H^{2}}{3 k^{3}}\left[k^{3}\left(\tau_{1}^{3}-\tau_{2}^{3}\right)+\mathcal{O}\left(k^{5} \tau_{i}^{5}\right)\right],
\end{aligned}
$$

where $k^{2} \tau_{i}^{2}$ indicate all possible combinations $k^{2} \tau_{1}^{2}, k^{2} \tau_{1} \tau_{2}$ and $k^{2} \tau_{2}^{2}$, and similarly for $k^{5} \tau_{i}^{5}$. Using the lowest order of these expansions, it is easy to count the powers of $\tau_{i}$ of the contribution of an arbitrary Feynman diagram. The $F$ two point function does not contribute any factor of $\tau_{i}$, and the $G^{R}$ two point function gives a factor of $\tau_{i}^{3}$. Furthermore, a vertex contributes a factor $a^{4}\left(\tau_{i}\right) \propto \tau_{i}^{-4}$, and an integral $\int d \tau_{i} \propto \tau_{i}$, so effectively it contributes a factor $\tau_{i}^{-3}$.

We can divide the Feynman diagrams into two classes: diagrams that contain only vertices with one dashed line (diagrams $\mathrm{A}, \mathrm{B}$ and $\mathrm{D}$ in the example of the two point function (2.33) $)$, and diagrams that contain one or more vertices with three dashed lines (diagram $\mathrm{C}$ in $(2.33)$ ). Because each dashed line is attached to a $G^{R}$ two point function, 
diagrams of the first class have an equal number of vertices as $G^{R}$ two point functions. Each vertex contributes a factor $\tau_{i}^{-3}$ and each $G^{R}$ two point function a factor of $\tau_{i}^{3}$ and therefore diagrams from the first class are proportional to $\tau_{i}^{0}$. Because these diagrams can contain integrals like $\int d \tau_{i} / \tau_{i}$, they can be proportional to powers of $\ln \left(-\tau_{i}\right)$, which are largest for the upper limit of the time integrals, i.e. $\tau$.

Diagrams from the second class have more $G^{R}$ two point functions than vertices, and are therefore proportional to positive powers of $\tau_{i}$. For example a diagram with one vertex with three dashed lines has two more $G^{R}$ two point functions than if this vertex would have had one dashed line, and is therefore suppressed by a factor of $\left|k \tau_{i}\right|^{6}$. The contributions from diagrams of the second class are largest for the lower limit of the time integrals, i.e. for $\tau_{H}$. Therefore the contributions of diagrams from this class are suppressed with respect to contributions of the first class by a factor of $\left|k \tau_{H}\right|^{6}=\exp \left(-6 N_{H}\right)$, and they do not have the growing factors of $\ln \left(\tau / \tau_{H}\right)$.

The power counting of $\tau_{i}$ that is done here, is similar to the power counting of the scale factor $a$ in the derivation of the theorem in 31]. The expansion of the two point functions (2.37) and (2.38) can be compared with the asymptotic expansions of the wavefunctions for late times in [31]. The difference is that here we use power counting to differentiate between growing and vanishing contributions to correlation functions for a specific $\left(\phi^{3}\right)$ interaction, whereas in [31] it was used to differentiate between interactions leading to different late time behaviour. We return to this in section 5.2.

\subsubsection{Large internal momenta}

We consider an arbitrary amputated 1PI diagram, which has small external momenta, and where we integrate the internal momenta starting at a scale $M$ somewhat smaller than $H$. We use a cutoff $\Lambda$ as ultraviolet regulator. Because both $M$ and $\Lambda$ are physical scales and not comoving scales, the limits of the momentum integrals are time dependent: $M a\left(\tau_{i}\right)$ and $\Lambda a\left(\tau_{i}\right)$, where $\tau_{i}$ corresponds with the time of one of the vertices. We take for this time the earliest time that occurs in the loop, because that corresponds with the smallest cutoff. ${ }^{3}$ In this way, the momentum integrals do also contribute time dependencies, which we also have to take into account.

Suppose that our arbitrary amputated 1PI diagram has $E$ external lines and $N$ vertices, each with $V$ legs. Then there are $P$ internal lines and $L$ loops with

$$
\begin{aligned}
P & =\frac{1}{2}(N V-E), \\
L & =\frac{1}{2}(N V-E)-N+1 .
\end{aligned}
$$

Furthermore from equations (2.24) and (2.25) we see that each internal two point function contributes factors proportional to

$$
\frac{\left(p_{i} \tau_{j_{1}}\right)^{n_{\beta}}}{p_{i}^{3}} e^{ \pm i p_{i} \tau_{j_{2}}}
$$

\footnotetext{
${ }^{3}$ If an ultraviolet divergence is local, it occurs only if the times of the vertices in the loop are equal (i.e. they are proportional to $\delta\left(\tau_{j_{1}}-\tau_{j_{2}}\right)$ for all times $\left.\tau_{j_{i}}\right)$; then it does not matter which time one chooses.
} 
where $n_{\beta}=0,1,2$. Each vertex gives $\int d \tau_{j} / \tau_{j}^{4}$, and each loop gives an integral $\int d^{3} p_{i}$. We ignore powers of the external momenta because the internal momenta are much larger, $p \gg k$. Then we can count the powers of $p$ and $\tau$ of the diagram:

$$
\left.p_{i}^{(-3+n) P+3 L-l} \tau_{j}^{-3 N+n P-l}\right|_{M a\left(\tau_{j}\right)} ^{\Lambda a\left(\tau_{j}\right)} \rightarrow\left(\frac{\Lambda}{H}\right)^{\frac{n}{2}(N V-E)-3 N-l+3} \tau_{j}^{-3},
$$

where $n$ is the sum of the $n_{\beta}$, and where $l$ is a non-negative integer that represents the fact that $p$ integrals can also lead to factors of $1 / \tau$ instead of an extra factor $p$ (see e.g. the integrals $(\overline{D .22})-(\overline{D .30})$ in the example calculation of Appendix D). We ignore the contribution from the lower limit $M a\left(\tau_{j}\right)$, because the full result cannot depend on the split in the integrals. ${ }^{4}$

Apparently the power of $\tau_{j}$ is independent of the details of the calculation: contributions from large internal momenta to 1 PI diagrams are always proportional to $\tau_{j}^{-3}$.

We can now compare the 1PI diagram, seen as an effective coupling, with a tree level coupling. They are both proportional to $\tau_{j}^{-3}$, but they differ in the possible numbers of (external) dashed lines. The 1PI diagrams can have any number of dashed lines, instead of one or three dashed lines for the tree level coupling. However, as shown in Appendix B, it turns out that 1PI diagrams with no external dashed lines vanish. Hence the non-vanishing 1PI diagrams can have one or more external dashed lines.

The 1PI diagrams, being effective couplings for small momenta, can be put in the analysis for small internal momenta of section 2.2.2. The power counting argument of that section shows that only those effective couplings with one external dashed line can lead to contributions proportional to $\tau^{0}$. Effective couplings with more external dashed lines lead to contributions that are suppressed by (at least) a factor $\left|k \tau_{H}\right|^{3}$.

The power of $\Lambda / H$ in equation (2.42) does depend on the details of the calculation. When it is non-negative, it can cause an ultraviolet divergence. Some divergent terms are proportional to $\delta\left(\tau_{j_{1}}-\tau_{j_{2}}\right)$ for all the times $\tau_{j_{i}}$ that occur in the loop. These divergences are the usual local divergences and are canceled by counterterms. Non-local divergent terms can also occur (an example of this is given below), as a consequence of the way in which the total correction is split up in contributions from individual diagrams. They must cancel between different contributions, in order to make the total result finite.

It is interesting to consider the errors that occur when the ultraviolet regulator $\Lambda$ is not taken to infinity but kept finite. From equation 2.42 it appears that, after the counterterms have been taken into account, the errors will be proportional to positive powers of $H / \Lambda$. Hence if $\Lambda$ is taken to be smaller than $H$, large errors occur, but if $\Lambda$ is taken to be larger than $H$ the errors are suppressed. Clearly, internal momenta of the order magnitude of the Hubble scale $H$ still contribute to the correlation functions, even though $H$ is much larger than the external momenta $k / a(\tau)$. This is a feature of quantum field theory in de Sitter space that is different from what one would expect from field theory in flat space. In the latter case, from the point of view of effective field theories, one expects only contributions from internal momenta of the order of magnitude of the

\footnotetext{
${ }^{4}$ This is confirmed for the specific case considered in Appendix D.
} 
external momenta. Contributions from higher scales are said to decouple. In de Sitter space, scales decouple only when they are larger than the Hubble scale $H$.

\subsubsection{Example: Late time contributions to equal time two point function at one loop}

The general analysis of this subsection can be checked in the example of the late time contributions to the one loop correction to the two point function (2.33), as calculated in Appendix D. In this calculation the momenta are split up between small and large at a comoving scale $M_{\mathrm{cm}}$, which obeys $\left|M_{\mathrm{cm}} \tau\right| \ll 1$ and $M_{\mathrm{cm}}>k$.

Small internal momenta. The results for small internal momenta of diagrams A and $\mathrm{B}$ are given in equations (D.11) and (D.58), respectively. Diagram C does not give any late time contribution. After attaching the external lines, the dominant terms can be found in equations (D.49) and (D.71) and are proportional to

$$
\frac{\lambda^{2}}{\epsilon} \ln ^{2} \frac{\tau}{\tau_{H}} \quad \text { with } \quad \epsilon=\frac{m^{2}}{3 H^{2}}, \quad \text { or } \quad \lambda^{2} \ln ^{3} \frac{\tau}{\tau_{H}},
$$

depending on the values of $\epsilon$ and $\ln \left(\tau / \tau_{H}\right)$, where $\epsilon$ is the infrared regulator. In this calculation we have used an expansion that is valid for $|\epsilon \ln (-k \tau)|<1$, and therefore this calculation is only valid for a limited amount of time. When $|\epsilon \ln (-k \tau)|$ approaches 1 , the term on the right in (2.43) becomes of comparable magnitude to the term on the left.

Two powers of the logarithm $\ln \left(\tau / \tau_{H}\right)$ come from the two time integrals corresponding with the two vertices. The extra factor of $\ln \left(\tau / \tau_{H}\right)$ in the term on the right in (2.43), is the consequence of the momentum integration, and was observed earlier in a similar calculation in [51], where also a small mass was used as infrared regulator.

Large internal momenta. For large momenta the result for the amputated diagrams are given in (D.42) for diagram A, (D.61) and (D.62) for diagram B and (D.65) for diagram C. Diagram A has a local ultraviolet divergence that is canceled by the counterterm, diagram D. Diagrams B and C have divergent terms that are non-local, and that cancel each other. The finite remainder is suppressed for late times. The contribution (D.62) from diagram B only removes the dependence on the scale $M_{\mathrm{cl}}$. Therefore only diagram A leads to late time contributions, in agreement with the result above that late time contributions can only come from 1PI diagrams with one external dashed line.

The term from diagram A that grows quickest for large internal momenta, after attaching the external lines, can be found in equation (D.49) and is proportional to

$$
\lambda^{2} \ln ^{3} \frac{\tau}{\tau_{H}}
$$

which is comparable to the term on the right in (2.43) for the small internal momenta.

Complete result for the one loop correction. The complete result of the late time contributions to the one loop correction is two times equation (D.49) added to equation 
(D.71), which gives

$$
\begin{gathered}
\frac{\lambda^{2}}{36(2 \pi)^{2} k^{3}}\left\{\frac{7}{9 \epsilon}+\frac{392}{27}-\frac{7}{3} \gamma-\frac{17}{18} \pi^{2}-\frac{4}{3} \ln 2-4 \zeta(3)-\ln \frac{2 \mu}{H}+\frac{4}{9} \ln \left(-k \tau_{H}\right)+\right. \\
\left(\frac{2}{\epsilon}+15-\frac{17}{3} \gamma-\frac{2}{3} \pi^{2}-\frac{8}{3} \ln 2-3 \ln \frac{2 \mu}{H}+\frac{8}{3} \ln \left(-k \tau_{H}\right)\right) \ln \frac{\tau}{\tau_{H}}+ \\
\left.\left(\frac{2}{\epsilon}+\frac{22}{3}-2 \gamma-2 \ln 2+4 \ln \left(-k \tau_{H}\right)\right) \ln ^{2} \frac{\tau}{\tau_{H}}+\frac{8}{3} \ln ^{3} \frac{\tau}{\tau_{H}}+\mathcal{O}\left(\frac{\tau}{\tau_{H}}\right)+\mathcal{O}(\epsilon)\right\} .
\end{gathered}
$$

Note that a consequence of the growing behaviour of loop corrections is that the theory become nonperturbative if one waits long enough.

\section{Classical theory}

In this section we consider classical $\phi^{3}$ theory for a massless minimally coupled field $\phi$. The evolution of classical fields on a de Sitter background is governed by the equation of motion (which can be derived from the Lagrangian density (2.1))

$$
\partial_{\tau}^{2} \phi(x)+2 H a(\tau) \partial_{\tau} \phi(x)-\nabla^{2} \phi(x)+a^{2}(\tau) \frac{\lambda}{2 !} \phi^{2}(x)=0,
$$

where we use $x=(\tau, \mathbf{x})$ with $\tau$ conformal time. Initial conditions have to be imposed at an initial time $\tau_{\text {in }}$. We focus on the calculation of equal time correlation functions

$$
\left\langle\phi\left(\tau, \mathbf{x}_{1}\right) \ldots \phi\left(\tau, \mathbf{x}_{n}\right)\right\rangle_{\mathrm{cl}}
$$

where the subscript "cl" denotes a correlation function in the classical theory. In this section we show how to calculate these correlation functions in a way that is similar to the interaction picture in quantum field theory: first we calculate the free field correlation functions starting from the initial conditions and using the free equations of motion, and then we calculate perturbative corrections, expressed in terms of these free field correlation functions. In 61, 57 this method was used in the context of thermal field theory. Furthermore, we show that the contributions to the correlation functions can be represented graphically in a way that is similar to Feynman diagrams.

\subsection{Perturbative calculation of correlation functions}

We assume that at the initial time $\tau_{\text {in }}$, initial conditions are given for the correlation functions

$$
\left\langle\phi\left(\tau_{\text {in }}, \mathbf{x}_{1}\right) \ldots \phi\left(\tau_{\text {in }}, \mathbf{x}_{n}\right)\right\rangle_{\mathrm{cl}}
$$

and first order time derivatives of these correlation functions. In the free field case $(\lambda=0)$, the initial conditions can be evolved in time using the free field equations of motion. Then one obtains the free field correlation functions

$$
\left\langle\phi_{0}\left(\tau_{1}, \mathbf{x}_{1}\right) \ldots \phi_{0}\left(\tau_{n}, \mathbf{x}_{n}\right)\right\rangle_{\mathrm{cl}}
$$

where the subscript " 0 " denotes the free field solutions, and where the times $\tau_{1}, \ldots, \tau_{n}$ do not have to be equal. 
To calculate perturbative corrections to the correlation functions, we first solve the classical equation of motion for $\phi(x)$ perturbatively. The first order correction is

$$
\phi_{1}(x)=-\frac{\lambda}{2 !} \int d^{4} y a^{4}\left(y_{0}\right) G^{R}(x, y) \phi_{0}^{2}(y)
$$

where $y_{0}$ denotes conformal time, and where the retarded propagator $G^{R}(x, y)$ is the solution of

$$
\frac{1}{a^{2}\left(x_{0}\right)}\left(\partial_{x_{0}}^{2}+2 H a\left(x_{0}\right) \partial_{x_{0}}-\nabla^{2}\right) G^{R}(x, y)=\frac{\delta^{4}(x-y)}{a^{4}\left(x_{0}\right)}
$$

where we used de Sitter metric (2.23). This equation is the same as (2.21), so that the retarded propagator in the classical theory is equal to the one in quantum theory, given in equation (2.25) after a spatial Fourier transform. Higher order perturbative corrections to the solution of the equation of motion are obtained by

$$
\phi_{i}(x)=-\frac{\lambda}{2 !} \int d^{4} y a^{4}\left(y_{0}\right) G^{R}(x, y) \sum_{j=0}^{i-1} \phi_{j}(y) \phi_{i-j-1}(y) .
$$

By iteration the $i$-th order solution can be expressed in terms of the zeroth order solution $\phi_{0}(x)$. The full perturbative solution of the equation of motion (3.1) is the sum

$$
\phi(x)=\sum_{i} \phi_{i}(x)
$$

Perturbative corrections to the correlation function (3.2) are obtained by replacing the $\phi\left(x_{i}\right)$ 's in (3.2) by the perturbative solution (3.8), and ordering the terms according to the total powers of $\lambda$ :

$$
\left\langle\phi\left(\tau, \mathbf{x}_{1}\right) \ldots \phi\left(\tau, \mathbf{x}_{n}\right)\right\rangle_{\mathrm{cl}}=\sum_{r}\left\langle\phi\left(\tau, \mathbf{x}_{1}\right) \ldots \phi\left(\tau, \mathbf{x}_{n}\right)\right\rangle_{\mathrm{cl}}^{r}
$$

with

$$
\left\langle\phi\left(\tau, \mathbf{x}_{1}\right) \ldots \phi\left(\tau, \mathbf{x}_{n}\right)\right\rangle_{\mathrm{cl}}^{r}=\sum_{i_{1}+\ldots+i_{n}=r}\left\langle\phi_{i_{1}}\left(\tau, \mathbf{x}_{1}\right) \ldots \phi_{i_{r}}\left(\tau, \mathbf{x}_{n}\right)\right\rangle_{\mathrm{cl}} .
$$

When the $\phi_{i_{j}}$ 's are completely expressed in terms of free field solutions $\phi_{0}(x)$, the corrections to the correlation function (3.10) are expressed in terms of free field correlation functions, which we have obtained from the free equations of motion and the initial conditions in (3.4).

\subsection{Graphical representation}

When we choose the initial conditions to be Gaussian, it is possible to represent the contributions on the right hand side of equation (3.10) graphically in a way that is similar to the Feynman diagrams of the quantum theory. In the free field case, Gaussian initial conditions evolve to Gaussian free field correlation functions. Therefore the free field correlation functions are completely determined by the two point function, which we call suggestively $F_{\text {cl }}$ :

$$
F_{\mathrm{cl}}\left(x_{1}, x_{2}\right)=\left\langle\phi_{0}\left(x_{1}\right) \phi_{0}\left(x_{2}\right)\right\rangle_{\mathrm{cl}}
$$


We assign graphical rules analogously to the quantum case:

$$
\begin{array}{ll}
\frac{x}{y}- & =-i G^{R}(x, y), \\
\frac{x \quad y}{2} & =F_{\mathrm{cl}}(x, y), \\
& =\frac{-i \lambda}{2} \int d^{4} y a^{4}\left(y_{0}\right),
\end{array}
$$

and furthermore

$$
\stackrel{x \rightarrow}{\times} \phi_{0}(x) .
$$

The $r$-th order corrections on the right-hand side of equation (3.10) can be constructed graphically in two steps. First the $\phi_{i}$ 's of equation (3.7) are represented by tree graphs where the endpoint $x$ and the $i$ vertices are connected to each other by $G^{R}$ propagators. The remaining free legs of the vertices are occupied by the $\phi_{0}$ 's of (3.15). For example the second order solution $\phi_{2}(x)$ can be represented by

$$
\begin{array}{r}
x-<_{\times}<_{\times}^{\times} \phi_{2}(x)=2 \int d^{4} y a^{4}\left(y_{0}\right)(-i) G^{R}(x, y) \frac{-i \lambda}{2 !} \phi_{0}(y) \times \\
\\
\int d^{4} z a^{4}\left(z_{0}\right)(-i) G^{R}(y, z) \frac{-i \lambda}{2 !} \phi_{0}^{2}(z),
\end{array}
$$

where the factor 2 comes from two equal contributions.

In the second step the tree graphs representing the $\phi_{i_{j}}$ in (3.10) are glued together at the crosses in all possible ways. When two crosses are glued together, a full line is created representing the free two point function (3.13). Consider for example the contribution $\left\langle\phi_{2}\left(x_{1}\right) \phi_{0}\left(x_{2}\right)\right\rangle_{\mathrm{cl}}$ to the second order two point function $\left\langle\phi\left(x_{1}\right) \phi\left(x_{2}\right)\right\rangle_{\mathrm{cl}}^{(2)}$. The tree graphs representing $\phi_{2}\left(x_{1}\right)$ and $\phi_{0}\left(x_{2}\right)$ can be glued together in two ways:

$$
\begin{aligned}
& x_{1}-\int^{x_{2}}(-i)^{2}(-i \lambda)^{2} \int d^{4} y a^{4}\left(y_{0}\right) G^{R}\left(x_{1}, y\right) \times \\
& \qquad d^{4} z a^{4}\left(z_{0}\right) G^{R}(y, z) F_{\mathrm{cl}}(y, z) F_{\mathrm{cl}}\left(z, x_{2}\right),
\end{aligned}
$$

(where an extra factor 2 comes from two ways of contracting the $\phi_{0}$ 's), and

$$
\begin{array}{r}
x_{1} \sum_{x_{2}} \frac{(-i)^{2}(-i \lambda)^{2}}{2} \int d^{4} y a^{4}\left(y_{0}\right) G^{R}\left(x_{1}, y\right) F_{\mathrm{cl}}\left(y, x_{2}\right) \times \\
\int d^{4} z a^{4}\left(z_{0}\right) G^{R}(y, z) F_{\mathrm{cl}}(z, z) .
\end{array}
$$

The former diagram is equal to diagram $\mathrm{A}$ in the quantum theory (equation (2.33), after a spatial Fourier transform) if $F_{\mathrm{cl}}\left(x_{1}, x_{2}\right)$ equals the $F$ two point function in equation (2.24). 
Both diagrams (3.17) and (3.18) can be divergent, depending on $F_{\mathrm{cl}}$. If the divergences are local they can be canceled by adding counterterms. In fact diagram (3.18) contains a tadpole diagram, which is automatically local and can be canceled completely by a linear counterterm.

Similarly one can construct the contribution $\left\langle\phi_{0}\left(x_{1}\right) \phi_{2}\left(x_{2}\right)\right\rangle_{\mathrm{cl}}$, which is equal to the mirror version of diagram $\mathrm{A}$ in (2.33). Finally there is $\left\langle\phi_{1}\left(x_{1}\right) \phi_{1}\left(x_{2}\right)\right\rangle_{\mathrm{cl}}$, which is equal to diagram $\mathrm{B}$ in the quantum theory. It is not possible to obtain diagram $\mathrm{C}$ in the classical theory.

Note that the resulting classical diagrams can have loops. This illustrates that loop corrections occur not only in the quantum theory, but also in the classical theory. These diagrams do not vanish, because there are statistical fluctuations.

\section{Classical approximation}

In section 2 we have investigated the late time behaviour of the quantum theory, and in section 3 we have set up the classical theory. The graphical representation of the classical perturbative corrections as described in section 3.2 suggests that the classical theory reproduces exactly the diagrams of the quantum theory with only vertices with one dashed line. In Appendix 9 a precise argument is given that shows that this is indeed the case. Hence if we choose the initial conditions of the classical approximation such that the classical free field two point function $F_{\mathrm{cl}}$ is equal to the quantum $F$ two point function (2.24), the classical approximation reproduces the contributions of the quantum theory coming from these diagrams.

In the one loop correction to the two point function (2.33), this means that the classical approximation is given by diagrams A, B and D (counterterms are still necessary in the classical approximation).

In this section we investigate how good the classical theory is as a classical approximation to the quantum theory for late times.

\subsection{Small internal momenta}

As argued in section 2.2.2 for small internal momenta, the diagrams with only vertices with one dashed line give exactly the contributions in the quantum theory that are proportional to $\tau^{0}$. The other diagrams, that have vertices with three dashed lines and are not in the classical approximation, give contributions that are suppressed by $\left|k \tau_{H}\right|^{6}=\exp \left(-6 N_{H}\right)$, because each vertex with three dashed lines leads to two more retarded propagators compared to a vertex with one dashed line. These contributions do not grow after horizon exit. Therefore, for small internal momenta the classical approximation is good up to errors that are suppressed by a factor of $\exp \left(-6 N_{H}\right)$ with respect to the late time contributions.

This is confirmed in the example of the one loop correction to the two point function. Here the late time contributions from small internal momenta are completely coming from diagrams $\mathrm{A}$ and $\mathrm{B}$ and these diagrams do indeed occur in the classical approximation.

The internal momenta in tree diagrams are always small. Therefore the tree level contributions in the quantum theory can well be approximated by a classical approximation, 
if $\tau_{H}$ is chosen sufficiently long after horizon exit. Note that this is not trivial: the quantum theory contains tree diagrams with vertices with three dashed lines that do not occur in the classical approximation.

\subsection{Large internal momenta}

In section 2.2.3 we have seen that in the quantum theory, loop corrections get late time contributions from internal momenta up to the Hubble scale $H$, from 1PI diagrams with one external dashed line. This set of diagrams is not the same as the diagrams of the classical approximation. Namely, the 1PI diagrams with one external dashed line can contain vertices with three dashed lines, but these diagrams do not occur in the classical approximation. Therefore, the classical approximation misses late time contributions from large internal momenta. This could have been expected: the classical approximation is not supposed to be good for physics at scales around $H$.

For one loop corrections, the classical approximation can be saved: it turns out that the classical approximation does not miss any late time contributions, because at one loop level there are no 1PI diagrams having both one external dashed line and a vertex with three dashed lines. However, another problem arises: because the classical approximation has fewer diagrams than the quantum theory, not all ultraviolet divergences are canceled. In the next subsection, we treat these new ultraviolet divergences by introducing a cutoff.

In the two point function, this becomes apparent by the fact that the classical approximation does not contain diagram $\mathrm{C}$. As a consequence, the ultraviolet divergence of diagram $\mathrm{B}$ is not canceled. As mentioned above, this ultraviolet divergence is not local, and can therefore not be canceled by a counterterm.

\subsection{Classical approximation at one loop}

In order to deal with the ultraviolet divergences in the classical approximation at one loop, we introduce a cutoff at a physical scale $\Lambda$ (we use a physical scale and not a comoving scale, such that we can use the arguments of section 2.2.3). In the following we estimate the magnitude of the error that is induced by this cutoff, in one loop diagrams.

First of all there are the ultraviolet divergences that made the cutoff necessary. From equation (2.42) we see that they are proportional to $(\Lambda / H)^{n_{1}}$ with $n_{1} \geq 0$ (the case $n_{1}=0$ can give a factor $\ln (\Lambda / H))$. As argued above, these divergences occur (at least at one loop level) only in 1PI diagrams with two or more external dashed lines, and are therefore, according to the argument in section 2.2.3, suppressed by a factor $\exp \left(-3 N_{H}\right)$ or a positive power of this factor. Thus errors coming from the divergent terms have an extra factor of $\exp \left(-3 N_{H}\right)(\Lambda / H)^{n_{1}}$ with respect to late time contributions from large internal momenta.

This can be checked for the example in Appendix D: the term of the amputated version of diagram $\mathrm{B}$ that causes a linear divergence in the classical approximation is given in (D.61). Together with the external $G^{R}$ two point functions, the divergent term has an extra factor $\left|k \tau_{H}\right|^{3}(\Lambda / H)$ with respect to non-vanishing late time contributions, e.g. equation (D.62).

Secondly there are the errors from terms that are proportional to inverse powers of $\Lambda$ and that would vanish if the cutoff would be sent to infinity. For 1PI diagrams with one 
external dashed line, these errors can be proportional to $\tau^{0}$ and give late time contributions. These errors therefore have only an extra factor of $(H / \Lambda)^{n_{2}}$ with $n_{2}>0$, with respect to other late time contributions from large internal momenta.

The total error thus has scales like

$$
c_{1} e^{-3 N_{H}}\left(\frac{\Lambda}{H}\right)^{n_{1}}+c_{2}\left(\frac{H}{\Lambda}\right)^{n_{2}}
$$

with respect to other late time contributions, where $c_{i}$ are constants of order $\mathcal{O}(1)$. To make this factor considerably smaller than one, $\tau_{H}$ should be chosen long enough after horizon exit and the cutoff $\Lambda$ should be chosen considerably larger than the Hubble scale $H$; e.g. if $n_{1}=n_{2}=1$ and $c_{1}=c_{2}, \Lambda$ should be chosen of the order of $\exp \left(3 N_{H} / 2\right) H$.

Instead of introducing the cutoff by hand in the momentum integrals, one can also remove the ultraviolet divergences by changing the ultraviolet behaviour of the initial conditions. For example one can put a cutoff in the initial conditions:

$$
\int d^{3} x e^{-i \mathbf{k} \cdot \mathbf{x}}\left\langle\phi\left(\tau_{H}, \mathbf{x}\right) \phi\left(\tau_{H}, \mathbf{0}\right)\right\rangle_{\mathrm{cl}}=\frac{H^{2}}{2 k^{3}}\left(1+k^{2} \tau_{H}^{2}\right) \theta\left(\Lambda_{\mathrm{cm}}-k\right),
$$

where $\Lambda_{\mathrm{cm}}$ is now a cutoff in comoving momentum. The physical scale $\Lambda$ that corresponds with $\Lambda_{\mathrm{cm}}$ is $\left|\Lambda_{\mathrm{cm}} / a(\tau)\right|$, thus the error estimate (4.1) becomes now

$$
c_{1} e^{-3 N_{H}}\left|\Lambda_{\mathrm{cm}} \tau\right|^{n_{1}}+c_{2}\left|\frac{1}{\Lambda_{\mathrm{cm}} \tau}\right|^{n_{2}} .
$$

Another possibility is to choose initial conditions as

$$
\int d^{3} x e^{-i \mathbf{k} \cdot \mathbf{x}}\left\langle\phi\left(\tau_{H}, \mathbf{x}\right) \phi\left(\tau_{H}, \mathbf{0}\right)\right\rangle_{\mathrm{cl}}=\frac{H^{2}}{2 k^{3}} \frac{1+k^{2} \tau_{H}^{2}}{1+k^{2} \tau_{c}^{2}},
$$

where there is not a hard cutoff, but the loop diagrams are made finite (except for the tadpole diagram, which does not cause problems because it can be canceled by a local counterterm). The time $\tau_{c}$ acts like an inverse (soft) cutoff in comoving momenta, so that it should be taken small enough to make $\tau / \tau_{c} \gg 1$ and large enough to keep $\left|k \tau_{H}\right|^{3}\left(\tau / \tau_{c}\right)^{n}$ small.

\subsection{Example}

In Appendix D we have calculated the one loop correction to the two point function in the quantum theory. As an example, we have computed numerically the error that comes from using a finite cutoff for a specific set of parameters, as a function of the cutoff. This is done for diagram A by taking the upper limit in equation (D.31), adding the counterterm (diagram D), and subtracting the term that remains finite in the limit $\Lambda \rightarrow \infty$ (i.e. the second line of $(\overline{\mathrm{D} .42}))$. Then the external lines are attached and the times $\tau_{1}$ and $\tau_{2}$ are integrated numerically. For diagram B the upper limit in equation (D.60) is used, and there are no counterterms. The results are divided by the full correction (2.45), and are

plotted, separately for diagrams A and D, and for diagram B, in figure 1. Both errors are much smaller than the full correction (2.45). The error from diagrams A and D is clearly decreasing for increasing cutoff. The error from diagram B is suppressed, but increasing linearly with the cutoff. These results agree with the arguments given in this section. 


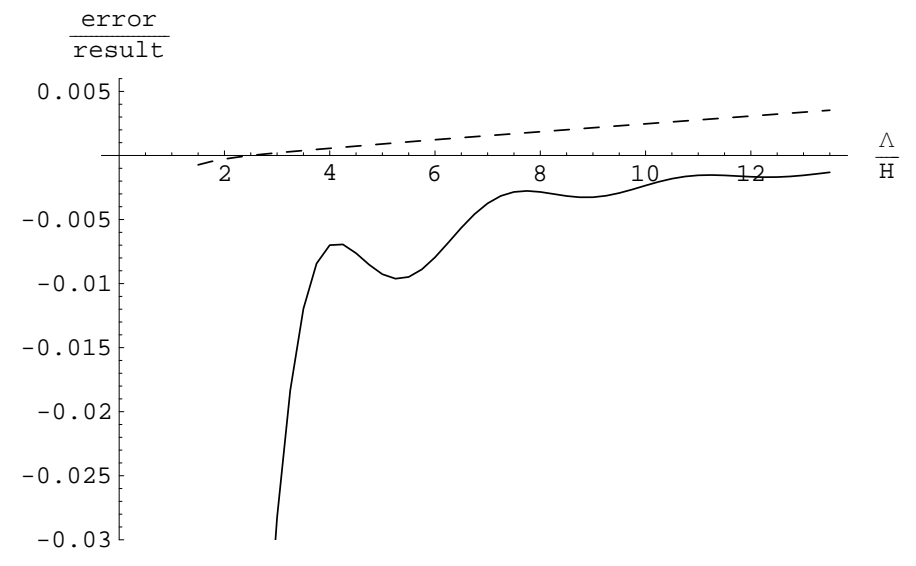

Figure 1: Plot of the numerically calculated error with respect to the complete result (2.45), for diagrams A and D (full) and diagram B (dashed), versus the cutoff $\Lambda / H$. We have used $k \tau_{H}=-0.4$, $k \tau=-0.03, \epsilon=0.1$, and $2 \mu=H$.

\section{Discussion and conclusions}

\subsection{Early time contributions}

Both in the quantum theory and in the classical theory we have neglected early time contributions, i.e. contributions from times before $\tau_{H}$. In both cases they can be included by imposing initial conditions at $\tau_{H}$, which can be obtained by calculating equal time correlation functions at $\tau_{H}$ in the quantum theory with initial time $\tau_{\text {in }} \rightarrow-\infty$.

In the quantum theory the initial conditions can be represented by non-local $n$-point vertex functions that act only at the initial time $\tau_{H}$, as explained for example in [30]. These vertex functions can have any number of dashed and full lines. They can give by themselves constant late time contributions, and can also occur in diagrams that give growing late time contributions. These extra ingredients make the arguments and calculations more complicated, but do not change them qualitatively. For example the calculation of the one loop correction to the two point function in Appendix D will have extra contributions from early times, of order $\lambda^{2}$ but without growing factors $\ln \left(\tau / \tau_{H}\right)$.

In the classical theory a similar thing can be done. A practical problem is that not all the vertex functions can be represented in the classical theory, because they can have any number of dashed lines, while in the classical theory only vertices with one dashed line can be represented. Instead the initial conditions at $\tau_{H}$ can be imposed by adjusting the free field correlation functions. They are then in general non-Gaussian and cannot be represented only by the free field two point function (3.11). As a consequence, the calculations become more complicated, but not qualitatively different, as is the case in the quantum theory.

\subsection{Generalization to derivative interactions}

Throughout this paper we have used $\phi^{3}$ theory as a toy model. For other interactions the analysis of this paper can be adjusted, which is straightforward for $\phi^{n}$ interactions, but 
less so for derivative interactions, which are of particular interest for cosmology.

Let us first consider contributions from small internal momenta. A spatial derivative leads to an inverse power of the scale factor, or equivalently to a factor of $\tau$. As can be seen from the power counting argument in section 2.2.2, this extra factor of $\tau$ suppresses late time contributions and prevents that any factors of $\ln \left(\tau / \tau_{H}\right)$ occur.

For a temporal derivative the situation is more complicated. If a time derivative $\partial_{t_{1}}=-H \tau_{1} \partial_{\tau_{1}}$ acts on an $F$ two point function, which can be expanded as (2.37), the constant term vanishes, so the result is proportional to $\tau_{i}^{2}$ and as a consequence, late time contributions are suppressed. But if a time derivative acts on a $G^{R}$ two point function, which can be expanded as (2.38), the result is still proportional to $\tau_{i}^{3}$, and there is no suppression of late time contributions yet. Only when there is also a time derivative acting on the other time argument $\partial_{t_{2}}$, the result becomes proportional to $\tau_{i}^{5}$ and late time contributions are suppressed.

Hence when an interaction has one time derivative, e.g. $\dot{\phi} \phi^{2}$, there are still late time contributions: if at all vertices (with one dashed line) the time derivatives act at the dashed line, no extra factors of $\tau_{i}$ appear and late time contributions are not suppressed. But when an interaction has two time derivatives, e.g. $\dot{\phi}^{2} \phi$, there is no way to avoid suppression of the late time contributions: in a diagram with $N$ vertices (all of this type and with one dashed line), there are $N G^{R}$ two point functions and $2 N$ time derivatives, so that there must be at least one $G^{R}$ two point function with time derivatives on both sides, or an $F$ two point function with a time derivative.

For large internal momenta, we can reconsider the power counting argument in section 2.2.3. A derivative (spatial or temporal) leads in (2.42) to either an additional factor $p \tau$, or to no additional factors. Hence derivatives only change the value of the integer $l$, and do not change the arguments of section 2.2.3. Apparently late time contributions from large internal momenta are not necessarily suppressed by derivative interactions.

In [31 these matters are treated in a slightly different way. There the time integrals are performed first, and after that the momentum integrals, which is a different order than employed in this paper. For fixed external and internal momenta, a theorem is derived that shows that if the interactions obey certain conditions, the time integrals converge for $\tau \rightarrow 0$. For the wavefunctions an asymptotic expansion is used, valid for late times (or equivalently small momenta).

Because in this theorem $\tau \rightarrow 0$ is taken, relative to which all fixed momenta are small, and because of the use of the asymptotic expansion, this theorem can be compared with our findings above for small internal momenta. They are indeed in agreement with the conditions of the theorem.

\subsection{Comparison to stochastic approach}

It is interesting to compare the classical theory as described in section 3, to the stochastic approach [62, 63]. In this approach the field $\phi$ is also considered to be a classical field with statistical fluctuations after horizon exit. The difference with the classical theory of section

3 is that the fluctuations are not imposed as initial conditions at a fixed initial time, but are put into the system stochastically at wavenumber $H$ at all times. 
The stochastic approach has in the recent literature been used for different purposes, e.g. for calculating non-Gaussianities perturbatively in multifield inflation [8, 9, 10, 11], and for investigating nonperturbative behaviour in de Sitter space that occurs at very late times [64, 65], when the factors $\ln (-\tau)$ have grown so large that they overcome the suppression by small coupling constants. In the latter case it has been argued [64, 65] that the stochastic approach can reproduce the terms with the largest power of $\ln (-\tau)$ at each order in the coupling constant (leading log approximation).

Below we first discuss the case of only a massless minimally coupled scalar field with nonderivative interactions, as in section 3. Then we make some remarks on theories with derivative interactions and with other fields than massless minimally coupled scalars.

\subsubsection{Massless minimally coupled scalar with nonderivative interactions}

The stochastic approach does not use a mass as infrared regulator, but uses a finite lower limit for the momentum integrals: the classical field $\phi$ is defined to contain only modes with comoving wavenumber $k>H$. Physically this corresponds to considering only a finite patch of de Sitter space, the size of which increases exponentially by the expansion. So to compare with the classical theory of section 3 , we need to reformulate the latter using this infrared regulator.

Apart from the different infrared regulator, the stochastic approach makes two additional approximations with respect to the classical theory of section 3. First the classical field is assumed not to contain modes with wavenumber $k>H a$; hence the momentum integrals have an upper limit $\mathrm{Ha}$. Second, the wavefunction of the free scalar field (A.4), that is used to characterize the stochastic fluctuations, is approximated by its leading term

for late times: $\phi_{k, 1}(\tau) \rightarrow i \frac{H}{\sqrt{2 k^{3}}}$. In the classical theory of section 3 this is equivalent to taking only the leading term of the expansion of the $F$ two point function, as is done in equation (2.37). Also the retarded propagator $G^{R}$ is approximated by its leading term, as in equation (2.38).

We now compare the stochastic approach with the classical theory of section 3 for the one loop correction of the two point function in $\phi^{3}$ theory, in particular the term with the largest power of $\ln (-\tau)$. For the classical theory we can use the calculation in Appendix $\mathrm{D}$; we only adjust the infrared regulator. This means that in the calculations for small internal momenta we put $\epsilon \rightarrow 0$, and use a lower limit $H$ for the momentum integrals. For diagram A this changes equation (D.11) to

$$
\frac{i \lambda^{2} \theta\left(\tau_{1}-\tau_{2}\right)}{6(2 \pi)^{2} H^{4}\left(\tau_{1} \tau_{2}\right)^{4}}\left(2\left(\tau_{1}^{3}-\tau_{2}^{3}\right) \ln \frac{M_{\mathrm{cm}}}{H}\right)
$$

The calculation for large internal momenta is unchanged and gives a finite contribution after adding the counterterm of diagram D. Attaching the external lines and performing the time integrals gives for the term with the largest power of $\ln (-\tau)$

$$
\frac{\lambda^{2}}{36(2 \pi)^{2} k^{3}}\left\{-\frac{1}{3} \ln ^{3} \frac{\tau}{\tau_{H}}\right\} \text {. }
$$


For the small internal momenta of diagram B we use the integral

$$
\int_{H}^{M_{\mathrm{cm}}} \frac{d p}{p^{2}} \int_{|p-k|}^{p+k} \frac{d p^{\prime}}{p^{\prime 2}}=\frac{1}{k^{2}}\left(\ln \frac{k^{2}}{H^{2}}+\ln \frac{M_{\mathrm{cm}}-k}{M_{\mathrm{cm}}+k}+\frac{2 k}{M_{\mathrm{cm}}}-2\right),
$$

so that the analog of (D.58) becomes

$$
\frac{-\lambda^{2}}{4(2 \pi)^{2} k^{3} H^{4}\left(\tau_{1} \tau_{2}\right)^{4}}\left(\ln \frac{k^{2}}{H^{2}}+\ln \frac{M_{\mathrm{cm}}-k}{M_{\mathrm{cm}}+k}+\frac{2 k}{M_{\mathrm{cm}}}-2\right) .
$$

Again the integral for the large internal momenta remains unchanged (we use a cutoff as described in section 4.3), and attaching the external lines and performing the time integrals gives a leading logarithmic term of $\ln ^{2}\left(\tau / \tau_{H}\right)$. Apparently there is no contribution to the $\ln ^{3} \tau / \tau_{H}$ term from diagram B when we use this infrared regularization.

We can calculate the same quantity in the stochastic approach by using stochastic sources. However we will not do this, but instead repeat the calculation of above, using the additional assumptions of the stochastic approach (i.e. taking the leading order approximations for the propagators (2.37), (2.38), and the upper limit $k<H a$ in the momentum integral). We expect that this does not make a difference for the result of the largest power of $\ln (-\tau)$ and therefore we interpret the result of this calculation as the result of the stochastic approach.

With the approximations of the stochastic approach, the calculation of diagram A reduces to the calculation for small internal momenta done above, with only the upper limit changed from $M_{\mathrm{cm}}$ to $\mathrm{Ha}\left(\tau_{2}\right)$. For the amputated version of diagram A the result is then

$$
\frac{i \lambda^{2} \theta\left(\tau_{1}-\tau_{2}\right)}{6(2 \pi)^{2} H^{4}\left(\tau_{1} \tau_{2}\right)^{4}}\left(2\left(\tau_{1}^{3}-\tau_{2}^{3}\right) \ln \frac{-1}{H \tau_{2}}\right)
$$

and after attaching the external lines and performing the time integrals, the result for the largest power of $\ln (-\tau)$ is the same as in our formulation of the classical theory, (5.2). For the amputated version of diagram $B$ the result is

$$
\frac{-\lambda^{2}}{4(2 \pi)^{2} k^{3} H^{4}\left(\tau_{1} \tau_{2}\right)^{4}}\left(\ln \frac{k^{2}}{H^{2}}+\ln \frac{H a\left(\tau_{2}\right)-k}{H a\left(\tau_{2}\right)+k}+\frac{2 k}{H a\left(\tau_{2}\right)}-2\right),
$$

which, similarly to the contribution (5.4) for our formulation of the classical theory, does not lead to $\ln ^{3} \tau / \tau_{H}$ terms.

In this calculation the stochastic approach reproduces the same leading logarithmic term (but not the same subleading logarithmic terms) as the classical theory in our formulation, using a lower momentum limit as infrared regulator. We remark that if we would have split the momentum integrals at a physical scale $M_{\mathrm{cm}} a$ instead of the comoving scale $M_{\mathrm{cm}}$ in the calculation of the classical theory, all the contributions to the leading logarithmic term would have come from small internal momenta. It is reasonable to expect that this remains true for higher orders. Moreover the approximations for the propagators in the stochastic approach are then also the same as we made in the calculation for the small internal momenta. Therefore we expect that the stochastic approach will give the largest logarithmic term at each order in the coupling, consistent with the arguments in [64, 65]. 
Because of the used approximations, the stochastic approach has fewer problems with the ultraviolet than our formulation of the classical theory (see sections 4.2 and 4.3). The drawback of using these approximations is that even at one loop order, only the leading logarithmic term can be obtained, whereas in our formulation of the classical theory, also the subleading logarithmic terms can be obtained at one loop order, as explained in section 4.3 .

\subsubsection{Derivative interactions and other fields}

As argued in section 5.2, derivative interactions typically lead to positive powers of $\tau$ and therefore to suppression of late time contributions from small internal momenta. There can also be interactions with fields that are not massless minimally coupled scalar fields. The wavefunctions of these fields are proportional to a positive power of $\tau$ for late times, instead of to $\tau^{0}$, as is the case for massless minimally coupled scalar fields. Therefore the leading terms of the expansions of the $F$ two point functions of these fields are also proportional to a positive power of $\tau$. If a diagram contains such an $F$ two point function, this diagram cannot lead to late time contributions from small internal momenta.

However, even if there are no late time contributions from small internal momenta, it is still possible that there are late time contributions from large internal momenta. The stochastic approach makes approximations that are not valid for large internal momenta. Therefore the stochastic approach can have problems with reproducing the largest logarithmic terms correctly in these cases. In [66] the stochastic approach is applied to the theory of a massless minimally coupled scalar field interacting with a massless fermion. Here the problem that there are no contributions from small internal momenta is circumvented by integrating out the fermion, and considering the effective theory of the scalar field.

\subsection{Application to the curvature perturbation}

The motivation for this work comes from cosmological perturbations generated during a period of inflation. As mentioned in the introduction, a suitable parameterization for these cosmological perturbations is the curvature perturbation $\zeta$, which typically has interactions involving derivatives. It is of interest to know late time contributions of correlation functions of $\zeta$, and whether these can be approximated by a classical approximation.

For small internal momenta, one can derive the interaction terms for the various degrees of freedom in a specific model of inflation, and then use the conditions of the theorem of [31] to decide whether these interactions can lead to late time contributions. For single field inflation (possibly together with $\mathcal{N}$ free massless scalar fields) it is shown in [31] that the interactions do obey the conditions, and therefore do not give late time contributions to all orders. This can be compared with [4], where it is argued, using classical physics, that $\zeta$ is conserved after horizon exit. This argument is only valid for adiabatic perturbations, which applies to single field inflation, and for small internal momenta, because the large internal momenta are removed by a smoothing procedure. Indeed, we found in section 4.1 that for small internal momenta the quantum theory can be approximated quite well by classical physics. 
For inflation models involving more fields, there are typically interactions that do not obey the conditions of the theorem in [31], and therefore can lead to late time contributions. Correspondingly, the perturbations are not adiabatic in these models, hence the argument of [4] does not apply and it is no surprise that $\zeta$ is not conserved after horizon exit.

For large internal momenta the situation is different. Since derivatives do not necessarily suppress late time contributions, it is possible that loop corrections lead to late time contributions, even for single field inflation. Moreover, a classical approximation would only be able to approximate these contributions up to one loop. An explicit calculation should decide on whether these contributions occur or not. The sample calculation in 31] seems to indicate that there are no late time contributions, even for large internal momenta, but we are concerned about the fact that in this calculation, $\tau \rightarrow 0$ is taken before the momentum integrals are performed. The terms that are discarded in this way, might lead to late time contributions.

Note that the background spacetime in inflation is not exactly de Sitter, but typically has a slowly decreasing Hubble constant $H$. This time dependence should be taken into account when deriving the wavefunctions for the fluctuating fields, and when doing the time integrals. Moreover, the fluctuations can react back on this background and in this way change the time dependence of $H$. This backreaction can be calculated by considering one point functions of the fluctuating fields, as is done for example in [67, 53, 54].

\subsection{Conclusions}

We have investigated up to which order corrections to cosmological correlation functions, generated after horizon exit, can be calculated reliably using classical physics. We have done this by making a detailed study of $\phi^{3}$ theory on a de Sitter background, for a massless minimally coupled scalar field $\phi$, as a toy model.

In the quantum theory we studied late time contributions (generated after horizon exit) to equal time correlation functions with external momenta much smaller than the Hubble scale. We found that in loop corrections, the loop integrals get contributions from internal momenta up to the Hubble scale $H$. This is different from the intuition from effective field theories in flat space, that loop integrals are dominated by internal momenta of the same order of magnitude as the external momenta. Our calculation of the one loop correction to the two point function supports the argument that the contributions from large internal momenta (around scale $H$ ) are not negligible: they are proportional to $\lambda^{2} \ln ^{3}\left(\tau / \tau_{H}\right)$, whereas the contributions from the small internal momenta are also proportional to $\lambda^{2} \ln ^{3}\left(\tau / \tau_{H}\right)$, or to $\lambda^{2} \ln ^{2}\left(\tau / \tau_{H}\right) / \epsilon$, with $\epsilon=m^{2} / 3 H^{2}$.

Furthermore we found that a classical approximation can approximate contributions from small internal momenta quite well, but that this does not hold for contributions from large internal momenta. This is not surprising, because the classical approximation is only supposed to work for physics at momentum scales much below the Hubble scale $H$. As a consequence, the classical approximation is good at tree level, but in general not for loop corrections. An exception is the one loop correction, for which the classical approximation can be good if an ultraviolet cutoff $\Lambda>H$ is introduced. 
We argued that the results presented for the $\phi^{3}$ toy model can be extended to derivative interactions, and be applied to the curvature perturbation $\zeta$. For non-Gaussian effects in multifield inflation models this means that at leading order, which is tree level, can be approximated quite well using classical physics. Also the one loop corrections can be approximated if a suitable cutoff is chosen. But for higher order corrections, the classical approximation is not expected to be good.

For small internal momenta, we found that derivatives tend to suppress late time corrections, in a way that agrees with the theorem derived by Weinberg [31. However, for large internal momenta, late time contributions need not to be suppressed. Consequently, it is possible that the curvature perturbation $\zeta$ is not conserved to all orders after horizon exit, even for single field inflation. There might be contributions to correlation functions of $\zeta$ that grow after horizon exit, coming from loop corrections. These contributions would be suppressed by powers of the coupling constant $H / M_{\mathrm{pl}}$ and possibly also by slow roll parameters, but they would be amplified by powers of the number of e-folds $\ln a$.

\section{Acknowledgments}

We thank Leo Kampmeijer, Jan Pieter van der Schaar and Koenraad Schalm for many useful discussions in a preliminary stage of this project. This work is supported by FOM/NWO.

\section{Note added}

Simultaneously with this work, [68] appeared on the arXiv, in which the stochastic approach is extended to scalar quantum electrodynamics. Similar as in [66] where the fermion is integrated out, here the vector field is integrated out and the resulting effective theory for the scalar is used for the stochastic approach.

\section{A. Free scalar field on a de Sitter background}

\section{A.1 Scalar wavefunction}

The free field equation of motion for a scalar field on a de Sitter background is

$$
\partial_{\tau}^{2} \phi(x)+2 H a(\tau) \partial_{\tau} \phi(x)-\nabla^{2} \phi(x)+a^{2}(\tau)\left(m^{2}+\xi R\right) \phi(x)=0,
$$

where we use $x=(\tau, \mathbf{x})$ with $\tau$ conformal time. After a spatial Fourier transformation the solutions for the mode functions are (see e.g. 69)

$$
\phi_{k, \alpha}(\tau)=-\frac{\sqrt{-\pi \tau}}{2 a(\tau)} H_{\nu}^{(\alpha)}(-k \tau)
$$

where $H_{\nu}^{(\alpha)}(-k \tau)$ are the Hankel functions for $\alpha=1,2$, and where $\nu$ is determined by

$$
\nu^{2}=\frac{9}{4}-\frac{m^{2}}{H^{2}}-12 \xi
$$


For massless minimally coupled fields, $m=0, \xi=0$ and we take $\nu=3 / 2$ (we choose $\nu$ to be positive). Then the modefunctions reduce to

$$
\phi_{k, 1}(\tau)=i \frac{H}{\sqrt{2 k^{3}}}(1+i k \tau) e^{-i k \tau}
$$

and $\phi_{k, 2}(\tau)=\phi_{k, 1}^{*}(\tau)$. For $k \tau \rightarrow-\infty$ the $\phi_{k, 1}(\tau)$ mode function is proportional to $e^{-i k \tau} / a(\tau)$ and is called the positive frequency solution ${ }^{5}$. Using these mode functions, the scalar field operator can be decomposed as

$$
\phi(\tau, \mathbf{x})=\int \frac{d^{3} k}{(2 \pi)^{3}}\left(e^{i \mathbf{k} \cdot \mathbf{x}} \alpha_{\mathbf{k}} \phi_{k, 1}(\tau)+e^{-i \mathbf{k} \cdot \mathbf{x}} \alpha_{\mathbf{k}}^{\dagger} \phi_{k, 1}^{*}(\tau)\right)
$$

where the annihilation operators $\alpha_{\mathbf{k}}$ and creation operators $\alpha_{\mathbf{k}}^{\dagger}$ satisfy the commutation relations

$$
\left[\alpha_{\mathbf{k}}, \alpha_{\mathbf{k}^{\prime}}^{\dagger}\right]=(2 \pi)^{3} \delta^{3}\left(\mathbf{k}-\mathbf{k}^{\prime}\right), \quad\left[\alpha_{\mathbf{k}}, \alpha_{\mathbf{k}^{\prime}}\right]=0 .
$$

The normalizations are chosen such that

$$
\left[\phi(\tau, \mathbf{x}), \pi\left(\tau, \mathbf{x}^{\prime}\right)\right]=i \delta^{3}\left(\mathbf{x}-\mathbf{x}^{\prime}\right)
$$

where $\pi(\tau, \mathbf{x})=a^{2}(\tau) \partial_{\tau} \phi(\tau, \mathbf{x})$ is the conjugate momentum, and that $a(\tau) \phi(\tau, \mathbf{x})$ is a conventionally normalized free field for $k \tau \rightarrow-\infty$. The state $|0\rangle$ defined by

$$
\alpha_{\mathbf{k}}|0\rangle=0
$$

corresponds therefore with the free vacuum state in Minkowski spacetime for $k \tau \rightarrow-\infty$. This state is called adiabatic or Bunch-Davies vacuum and is the state with respect to which we calculate correlation functions in this paper.

\section{A.2 Particle creation}

Despite the confusing nature of the concept of particle number in curved spacetime (see e.g. [69]), we give here a definition of the particle number in the frame of a comoving observer in de Sitter spacetime. This definition of particle number then allows us to make a comparison with the classical approximation in thermal field theory in the next subsection.

Using comoving time, the free Lagrangian is the spatial integral over the Lagrangian density (2.1) with $\lambda=0$

$$
L\left[\varphi_{\mathbf{k}}, \partial_{\tau} \varphi_{\mathbf{k}}\right]=\int \frac{d^{3} k}{(2 \pi)^{3}}\left(\frac{1}{2} a^{2}\left|\partial_{\tau} \varphi_{\mathbf{k}}\right|^{2}-\frac{1}{2} a^{2} k^{2}\left|\varphi_{\mathbf{k}}\right|^{2}-\frac{1}{2}\left(m^{2}+\xi R\right) a^{4}\left|\varphi_{\mathbf{k}}\right|^{2}\right),
$$

where we have taken

$$
\phi(\tau, \mathbf{x})=\int \frac{d^{3} k}{(2 \pi)^{3}} \varphi_{\mathbf{k}} e^{i \mathbf{k} \cdot \mathbf{x}}
$$

\footnotetext{
${ }^{5}$ Often the Hankel functions are used with a negative argument: $H_{\nu}^{(\alpha)}(k \tau)$. Then the $\phi_{k, 2}(\tau)$ mode function is the positive frequency solution.
} 
where the $\varphi_{\mathbf{k}}$ are time dependent operators. In this form the Lagrangian describes a system of uncoupled harmonic oscillators with frequencies $\omega_{k}=\sqrt{k^{2}+a^{2}\left(m^{2}+\xi R\right)}$. The conjugate momentum is defined as $\pi_{\mathbf{k}}=\delta L / \delta\left(\partial_{\tau} \varphi_{\mathbf{k}}\right)=a^{2} \partial_{\tau} \varphi_{\mathbf{k}}$, and using this the Hamiltonian becomes

$$
H\left[\varphi_{\mathbf{k}}, \pi_{\mathbf{k}}\right]=\int \frac{d^{3} k}{(2 \pi)^{3}}\left(\frac{1}{2} \frac{\left|\pi_{\mathbf{k}}\right|^{2}}{a^{2}}+\frac{1}{2} a^{2} k^{2}\left|\varphi_{\mathbf{k}}\right|^{2}+\frac{1}{2}\left(m^{2}+\xi R\right) a^{4}\left|\varphi_{\mathbf{k}}\right|^{2}\right) .
$$

By analogy to the harmonic oscillator, we define time dependent annihilation and creation operators $\bar{\alpha}_{\mathbf{k}}$ and $\bar{\alpha}_{\mathbf{k}}^{\dagger}$ by

$$
a \varphi_{\mathbf{k}}=\frac{1}{\sqrt{2 \omega_{k}}}\left(\bar{\alpha}_{\mathbf{k}} e^{-i k \tau}+\bar{\alpha}_{-\mathbf{k}}^{\dagger} e^{i k \tau}\right), \quad \frac{\pi_{\mathbf{k}}}{a}=\frac{1}{i} \sqrt{\frac{\omega_{k}}{2}}\left(\bar{\alpha}_{\mathbf{k}} e^{-i k \tau}-\bar{\alpha}_{-\mathbf{k}}^{\dagger} e^{i k \tau}\right) .
$$

They obey the commutation relation

$$
\left[\bar{\alpha}_{\mathbf{k}}, \bar{\alpha}_{\mathbf{k}^{\prime}}^{\dagger}\right]=(2 \pi)^{3} \delta^{3}\left(\mathbf{k}-\mathbf{k}^{\prime}\right)
$$

The Hamiltonian (A.11) expressed in terms of these operators has the familiar form

$$
H=\int \frac{d^{3} k}{(2 \pi)^{3}}\left(\bar{\alpha}_{\mathbf{k}}^{\dagger} \bar{\alpha}_{\mathbf{k}}+\frac{1}{2}\left[\bar{\alpha}_{\mathbf{k}}, \bar{\alpha}_{\mathbf{k}}^{\dagger}\right]\right) \omega_{k}
$$

If we take $m=0$ and $\xi=0$ we can use the modefunction $\phi_{k, 1}(\tau)$ of equation (A.4) to express the operators $\bar{\alpha}_{\mathbf{k}}, \bar{\alpha}_{\mathbf{k}}^{\dagger}$ in terms of the operators $\alpha_{\mathbf{k}}, \alpha_{\mathbf{k}}^{\dagger}$ in the mode decomposition A.5):

$$
\begin{aligned}
\bar{\alpha}_{\mathbf{k}} & =\frac{-i}{2 k \tau}(1+2 i k \tau) \alpha_{\mathbf{k}}+i \frac{e^{2 i k \tau}}{2 k \tau} \alpha_{-\mathbf{k}}^{\dagger}, \\
\bar{\alpha}_{-\mathbf{k}}^{\dagger} & =\frac{-i e^{-2 i k \tau}}{2 k \tau} \alpha_{\mathbf{k}}+\frac{i}{2 k \tau}(1-2 i k \tau) \alpha_{-\mathbf{k}}^{\dagger}
\end{aligned}
$$

For $k \tau \rightarrow-\infty$ the $\bar{\alpha}_{\mathbf{k}}$ operator becomes equal to $\alpha_{\mathbf{k}}$. We can define $n_{k}$ and $\tilde{n}_{k}$ by

$$
\begin{aligned}
\left\langle 0\left|\bar{\alpha}_{\mathbf{k}}^{\dagger} \bar{\alpha}_{\mathbf{k}^{\prime}}\right| 0\right\rangle & =n_{k}(2 \pi)^{3} \delta\left(\mathbf{k}-\mathbf{k}^{\prime}\right), & n_{k} & =\frac{1}{4 k^{2} \tau^{2}} \\
\left\langle 0\left|\bar{\alpha}_{\mathbf{k}} \bar{\alpha}_{-\mathbf{k}^{\prime}}\right| 0\right\rangle & =\left(\tilde{n}_{k}+\frac{i}{2 k \tau}\right)(2 \pi)^{3} \delta\left(\mathbf{k}-\mathbf{k}^{\prime}\right), & \tilde{n}_{k} & =\frac{1}{4 k^{2} \tau^{2}}
\end{aligned}
$$

where one can interpret $n_{k}$ as the particle number and $\tilde{n}_{k}$ as a kind of off-diagonal particle number, with respect to the vacuum at $k \tau \rightarrow-\infty$. Clearly these quantities are proportional to $a^{2}$.

\section{A.3 Comparison with thermal field theory}

It is interesting to compare with thermal field theory on a Minkowski background (see [61, 57]). The $F$ two point function in a thermal system with temperature $T$ in Minkowski spacetime is given by

$$
F\left(k, t_{1}, t_{2}\right)=\frac{1}{k}\left(n_{\mathrm{th}}(k)+\frac{1}{2}\right) \cos k\left(t_{1}-t_{2}\right) \quad n_{\mathrm{th}}(k)=\frac{1}{e^{k / T}-1},
$$


where $n_{\text {th }}(k)$ is the particle number. For small momenta with respect to the temperature $k \ll T, n_{\text {th }} \simeq T / k$, which becomes large and classical behaviour emerges. Moreover the $G^{R}$ two point function does not have this amplification factor; it is given by

$$
G^{R}\left(k, t_{1}, t_{2}\right)=\frac{\theta\left(t_{1}-t_{2}\right)}{k} \sin k\left(t_{1}-t_{2}\right) .
$$

Therefore a diagram containing a vertex with more than one dashed line is suppressed with respect to the same diagram with a vertex with one dashed line.

To compare this with the de Sitter case, it is natural to consider $a \phi_{k}(\tau)$. Then we have

$$
a\left(\tau_{1}\right) a\left(\tau_{2}\right) F\left(k, \tau_{1}, \tau_{2}\right)=\frac{1}{k}\left[\left(\frac{1}{2 k^{2} \tau_{1} \tau_{2}}+\frac{1}{2}\right) \cos k\left(\tau_{1}-\tau_{2}\right)+\frac{\tau_{1}-\tau_{2}}{2 k \tau_{1} \tau_{2}} \sin k\left(\tau_{1}-\tau_{2}\right)\right] .
$$

We see that for equal times $\tau_{1}=\tau_{2}=\tau$ this quantity grows as

$$
\frac{1}{2 k^{2} \tau^{2}}+\frac{1}{2} \propto n+\tilde{n}+\frac{1}{2}
$$

The quantity $a\left(\tau_{1}\right) a\left(\tau_{2}\right) G^{R}\left(k, \tau_{1}, \tau_{2}\right)$ does not have this amplification factor for late times, as can be seen from expansion for small $k \tau$. Therefore a diagram containing a vertex with more than one dashed line is suppressed with respect to the same diagram with a vertex with one dashed line, as in thermal field theory.

Both in the de Sitter case as in thermal field theory, the arguments given above explain why the classical approximation is good for small physical internal momenta, i.e. $k / a \ll H$ $(|k \tau| \ll 1)$ or $k \ll T$. But, as we argue in this paper for the de Sitter case, for large internal momenta $(\approx H$ or $\approx T)$ problems arise for the classical approximation, e.g. in the thermal case involving Hard Thermal Loops [70].

\section{B. Amputated diagrams with no external dashed lines}

The contribution of an amputated diagram with no external dashed lines, as for example occurs in diagram (2.34), vanishes. The reason is that in such a diagram there is always a closed loop of $G^{R}$ two point functions. This can be seen by picking an arbitrary vertex, and from there following one of the dashed lines, along the $G^{R}$ two point functions, from this vertex to a next vertex. From this new vertex one can repeat this to go to the next vertex. Because there is no external dashed line, this can be repeated indefinitely while staying inside the diagram. Since there is only a finite number of vertices in the diagram, one eventually ends up at a vertex for the second time. Therefore there must be a closed loop of $G^{R}$ two point functions in a diagram with no external dashed lines.

Because of the $\theta$-functions of the $G^{R}$ two point functions, the times of the vertices of a closed loop of $G^{R}$ two point functions have to be ordered. But in a closed loop of there is always at least one $G^{R}$ two point function for which the $\theta$-function vanishes, and therefore the complete diagram vanishes. Even if all the internal times of the loop are equal, the diagram vanishes because the $G^{R}$ two point function vanishes if the time arguments are equal. 


\section{Correspondence between diagrams in quantum and classical theory}

In this Appendix we show that, if one chooses initial conditions such that

$$
F_{\mathrm{cl}}\left(\mathbf{k}, \tau_{1}, \tau_{2}\right)=F\left(\mathbf{k}, \tau_{1}, \tau_{2}\right),
$$

the classical approximation reproduces the diagrams of the quantum theory with only vertices with one dashed line.

Discarding the vertex with three dashed lines in the quantum theory corresponds to discarding the term with $\left(\phi^{(2)}\right)^{3}$ in equation (2.15). An arbitrary equal time correlation function, calculated up to order $n$ in the coupling $\lambda$, can then be written as

$$
\left\langle\phi\left(\tau, \mathbf{x}_{1}\right) \ldots \phi\left(\tau, \mathbf{x}_{r}\right)\right\rangle=\left\langle\phi^{(1)}\left(\tau, \mathbf{x}_{1}\right) \ldots \phi^{(1)}\left(\tau, \mathbf{x}_{r}\right) \frac{1}{n !}\left(\bar{S}_{\mathrm{int}}\right)^{n}\right\rangle^{(0)},
$$

where the superscript (0) denotes the free correlation function, and where the modified interaction part of the action is given by

$$
\bar{S}_{\mathrm{int}}=-\int_{-\infty}^{\tau} d \tau a^{4}(\tau) \int d^{3} x \frac{\lambda}{2 !}\left(\phi^{(1)}\right)^{2} \phi^{(2)} .
$$

On the right hand side of equation (C.2) the fields $\phi^{(1)}$ and $\phi^{(2)}$ have to be contracted in all possible ways: every $\phi^{(2)}$ is contracted with a $\phi^{(1)}$ to a retarded propagator $G^{R}$, and the remaining $\phi^{(1)}$ 's are contracted with each other to $F$ two point functions. Suppose that we do have contracted the $\phi^{(2)}$ 's, but not yet the remaining $\phi^{(1)}$ 's. The correlation function can then be drawn as a number of disconnected diagrams, in which the vertices are connected by $G^{R}$ two point functions and which have loose ends representing $\phi^{(1)}$ 's that are yet to be connected. In total there are $n$ vertices. Each disconnected diagram can be a tree diagram or a loop diagram. A loop diagram with only $G^{R}$ two point functions vanishes, as explained in Appendix B. Hence if one of the disconnected diagrams contains a loop, the complete contraction does not contribute to the correlation function, and for all nonvanishing contractions the disconnected diagrams are tree diagrams. These tree diagrams are the same tree diagrams in the classical theory that represent the perturbative solutions $\phi_{i}$ in the classical theory (3.7), where all the $\phi^{(1)}$ 's in the quantum theory correspond with free field solutions $\phi_{0}$ in the classical theory. The symmetry factors are also equal because they arise in the same way (the factor $1 / n$ ! is canceled by a factor $n$ ! from the $n$ equivalent vertices). The remaining contractions of the $\phi^{(1)}$ 's in the quantum theory are equal to the contractions of the $\phi_{0}$ 's in the classical theory, because of equation (C.1).

Therefore the classical theory with the same couplings as the quantum theory, and using initial conditions such that (C.1) holds, gives all the diagrams using only the vertex with one dashed line in the quantum theory, up to vanishing diagrams. Hence this classical theory reproduces the late time contributions for small internal momenta.

\section{One loop correction to two point function}

In this appendix we calculate the one loop correction to the two point function as given in equation (2.33). We first consider the diagrams with one external dashed line (diagrams 
$\mathrm{A}$ and $\mathrm{D}$ ), and then the ones with two external dashed lines (diagrams B and C). The complete result is given in equation (2.45).

\section{D.1 Diagrams A and D}

We start with the diagrams with one external $G^{R}$ two point function. First we calculate the amputated diagrams, and then attach the external lines. The amputated diagrams are:

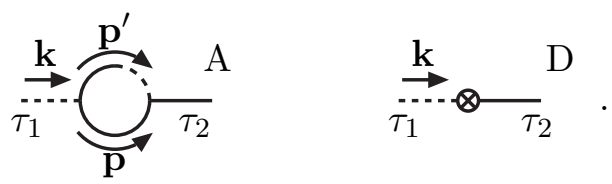

The amputated version of diagram $\mathrm{A}$ is given by

$$
\begin{array}{r}
\mathrm{A}_{\mathrm{amp}}\left(k, \tau_{1}, \tau_{2}\right)=\frac{-i(-i \lambda)^{2}}{H^{8} \tau_{1}^{4} \tau_{2}^{4}} \int \frac{d^{3} p d^{3} p^{\prime}}{(2 \pi)^{3}} \delta^{3}\left(\mathbf{k}-\mathbf{p}-\mathbf{p}^{\prime}\right) G^{R}\left(p^{\prime}, \tau_{1}, \tau_{2}\right) F\left(p, \tau_{1}, \tau_{2}\right)= \\
\frac{i \lambda^{2}}{(2 \pi)^{2} k H^{8} \tau_{1}^{4} \tau_{2}^{4}} \int_{0}^{\infty} d p p \int_{|p-k|}^{p+k} d p^{\prime} p^{\prime} G^{R}\left(p^{\prime}, \tau_{1}, \tau_{2}\right) F\left(p, \tau_{1}, \tau_{2}\right),
\end{array}
$$

where we have used the identity

$$
\int d^{3} p d^{3} p^{\prime} \delta^{3}\left(\mathbf{k}+\mathbf{p}+\mathbf{p}^{\prime}\right) f\left(k, p, p^{\prime}\right)=\frac{2 \pi}{k} \int_{0}^{\infty} d p p \int_{|p-k|}^{p+k} d p^{\prime} p^{\prime} f\left(k, p, p^{\prime}\right) .
$$

We will evaluate this integral below. For diagram D we see from equations $(2.30)$ and (2.3) that it is equal to

$$
\mathrm{D}_{\mathrm{amp}}\left(k, \tau_{1}, \tau_{2}\right)=-i a^{4}\left(\tau_{1}\right) \delta_{m} \delta\left(\tau_{1}-\tau_{2}\right)=\frac{-i \lambda^{2}}{4(2 \pi)^{2} H^{4} \tau_{1}^{4}} \ln \left(\frac{\Lambda}{\mu}\right) \delta\left(\tau_{1}-\tau_{2}\right),
$$

where $\Lambda$ is the ultraviolet momentum cutoff and $\mu$ is a renormalization scale. The counterterm $\delta_{Z}$ is finite and leads to terms proportional to positive powers of $\tau$, and is therefore left out.

We calculate the integral (D.2) by splitting the $p$ integral in a small momentum part $\int_{0}^{M_{\mathrm{cm}}} d p$ and a large momentum part $\int_{M_{\mathrm{cm}}}^{\Lambda a\left(\tau_{2}\right)} d p$, with $\left|M_{\mathrm{cm}} \tau_{i}\right| \ll 1$ and $M_{\mathrm{cm}}>k .{ }^{6}$

\section{D.1.1 Amputated diagram for small internal momenta}

The integral in equation (D.2) is infrared divergent for $p \rightarrow 0$. We regulate this divergence by giving the field a small mass $m \ll H$, such that $\nu=3 / 2-\epsilon$ with $\epsilon=m^{2} / 3 H^{2}$. The $F$ and $G^{R}$ two point functions are then, using equations (2.17), (2.18) and (A.5),

$$
\begin{aligned}
F\left(k, \tau_{1}, \tau_{2}\right) & =\frac{\pi \sqrt{\tau_{1} \tau_{2}}}{4 a\left(\tau_{1}\right) a\left(\tau_{2}\right)} \operatorname{Re}\left(H_{\nu}^{(1)}\left(-k \tau_{1}\right) H_{\nu}^{(1)^{*}}\left(-k \tau_{2}\right)\right) \\
G^{R}\left(k, \tau_{1}, \tau_{2}\right) & =-\frac{\pi \sqrt{\tau_{1} \tau_{2}}}{2 a\left(\tau_{1}\right) a\left(\tau_{2}\right)} \theta\left(\tau_{1}-\tau_{2}\right) \operatorname{Im}\left(H_{\nu}^{(1)}\left(-k \tau_{1}\right) H_{\nu}^{(1)^{*}}\left(-k \tau_{2}\right)\right) .
\end{aligned}
$$

\footnotetext{
${ }^{6}$ In [51] a similar split of integrals is used to calculate a similar integral. Note however that the integral there differs from the integral here, because the self-energy kernel of [51] is not the same as the amputated diagram A.
} 
Using (see [71])

$$
\begin{aligned}
H_{\nu}^{(1)}(-k \tau) & =J_{\nu}(-k \tau)+i\left(\frac{\cos \nu \pi}{\sin \nu \pi} J_{\nu}(-k \tau)-\frac{1}{\sin \nu \pi} J_{-\nu}(-k \tau)\right), \\
J_{\nu}(-k \tau) & =\frac{1}{\Gamma(\nu+1)}\left(-\frac{1}{2} k \tau\right)^{\nu}\left(1+\mathcal{O}\left(k^{2} \tau^{2}\right)\right),
\end{aligned}
$$

and the identity $\Gamma(\nu) \Gamma(1-\nu)=\pi / \sin \nu \pi$, we obtain

$$
\begin{aligned}
F\left(k, \tau_{1}, \tau_{2}\right) & =\frac{H^{2}}{2 k^{3}}\left(k^{2} \tau_{1} \tau_{2}\right)^{\epsilon} \\
G^{R}\left(k, \tau_{1}, \tau_{2}\right) & =\theta\left(\tau_{1}-\tau_{2}\right) \frac{H^{2}}{3}\left(\tau_{1}^{3}\left(\frac{\tau_{2}}{\tau_{1}}\right)^{\epsilon}-\left(\frac{\tau_{1}}{\tau_{2}}\right)^{\epsilon} \tau_{2}^{3}\right) .
\end{aligned}
$$

The integral is

$$
\begin{gathered}
\frac{i \lambda^{2}}{(2 \pi)^{2} k H^{8} \tau_{1}^{4} \tau_{2}^{4}} \frac{H^{4} \theta\left(\tau_{1}-\tau_{2}\right)\left(\tau_{1}^{3}\left|\tau_{2}\right|^{2 \epsilon}-\left|\tau_{1}\right|^{2 \epsilon} \tau_{2}^{3}\right)}{6} \int_{0}^{M_{\mathrm{cm}}} d p \frac{p^{2 \epsilon}}{p^{2}} \int_{|p-k|}^{p+k} d p^{\prime} p^{\prime}= \\
\frac{i \lambda^{2} \theta\left(\tau_{1}-\tau_{2}\right)\left(\tau_{1}^{3}\left|\tau_{2}\right|^{2 \epsilon}-\left|\tau_{1}\right|^{2 \epsilon} \tau_{2}^{3}\right)}{6(2 \pi)^{2} H^{4}\left(\tau_{1} \tau_{2}\right)^{4}} \frac{M_{\mathrm{cm}}{ }^{2 \epsilon}}{\epsilon}= \\
\frac{i \lambda^{2} \theta\left(\tau_{1}-\tau_{2}\right)}{6(2 \pi)^{2} H^{4}\left(\tau_{1} \tau_{2}\right)^{4}}\left(\frac{\tau_{1}^{3}-\tau_{2}^{3}}{\epsilon}+2 \tau_{1}^{3} \ln \left|M_{\mathrm{cm}} \tau_{2}\right|-2 \tau_{2}^{3} \ln \left|M_{\mathrm{cm}} \tau_{1}\right|+\mathcal{O}(\epsilon)\right) .
\end{gathered}
$$

\section{D.1.2 Amputated diagrams for large internal momenta}

For large momenta we approximate the field to be massless and we use the two point functions of equations (2.24) and (2.25), which we write as

$$
\begin{aligned}
F\left(k, \tau_{1}, \tau_{2}\right) & =\frac{H^{2}}{2} \sum_{i=1}^{3} F_{i}\left(k, \tau_{1}, \tau_{2}\right), \\
F_{1}\left(k, \tau_{1}, \tau_{2}\right) & =\frac{1}{k^{3}} \cos k \Delta \tau, \\
F_{2}\left(k, \tau_{1}, \tau_{2}\right) & =\frac{1}{k^{2}} \Delta \tau \sin k \Delta \tau, \\
F_{3}\left(k, \tau_{1}, \tau_{2}\right) & =\frac{1}{k} \tau_{1} \tau_{2} \cos k \Delta \tau,
\end{aligned}
$$

with $\Delta \tau=\tau_{1}-\tau_{2}$, and similarly

$$
\begin{aligned}
G^{R}\left(k, \tau_{1}, \tau_{2}\right) & =\theta\left(\tau_{1}-\tau_{2}\right) H^{2} \sum_{i=1}^{3} G_{i}^{R}\left(k, \tau_{1}, \tau_{2}\right), \\
G_{1}^{R}\left(k, \tau_{1}, \tau_{2}\right) & =\frac{1}{k^{3}} \sin k \Delta \tau, \\
G_{2}^{R}\left(k, \tau_{1}, \tau_{2}\right) & =\frac{-1}{k^{2}} \Delta \tau \cos k \Delta \tau, \\
G_{3}^{R}\left(k, \tau_{1}, \tau_{2}\right) & =\frac{1}{k} \tau_{1} \tau_{2} \sin k \Delta \tau .
\end{aligned}
$$


In the following calculations we use the definitions

$$
\operatorname{Si}(x)=\int_{0}^{x} d x^{\prime} \frac{\sin x^{\prime}}{x^{\prime}}, \quad \operatorname{Ci}(x)=-\int_{x}^{\infty} d x^{\prime} \frac{\cos x^{\prime}}{x^{\prime}},
$$

which behave for small respectively large arguments as

$$
\begin{array}{lrl}
\operatorname{Si}(x)=x+\mathcal{O}\left(x^{3}\right), & \operatorname{Si}(x)=\frac{\pi}{2}-\frac{\cos x}{x}-\frac{\sin x}{x^{2}}+\mathcal{O}\left(x^{-3}\right), \\
\mathrm{Ci}(x)=\gamma+\ln x-\frac{x^{2}}{4}+\mathcal{O}\left(x^{4}\right), & \mathrm{Ci}(x)=\frac{\sin x}{x}-\frac{\cos x}{x^{2}}+\mathcal{O}\left(x^{-2}\right),
\end{array}
$$

and the identities

$$
\begin{aligned}
& \int_{p-k}^{p+k} d p^{\prime} \frac{\sin p^{\prime} \Delta \tau}{p^{\prime 2}}=-\frac{\sin (p+k) \Delta \tau}{p+k}+\frac{\sin (p-k) \Delta \tau}{p-k}+ \\
& \Delta \tau(\operatorname{Ci}((p+k) \Delta \tau)-\operatorname{Ci}((p-k) \Delta \tau)),-\Delta \tau(\operatorname{Si}((p+k) \Delta \tau)-\operatorname{Si}((p-k) \Delta \tau)), \\
& \int_{p-k}^{p+k} d p^{\prime} \frac{\cos p^{\prime} \Delta \tau}{p^{\prime 2}}=-\frac{\cos (p+k) \Delta \tau}{p+k}+\frac{\cos (p-k) \Delta \tau}{p-k}+ \\
& \int_{p-k}^{p+k} d p^{\prime} \sin p^{\prime} \Delta \tau=\frac{-1}{\Delta \tau}(\cos (p+k) \Delta \tau-\cos (p-k) \Delta \tau)=\frac{2}{\Delta \tau} \sin k \Delta \tau \sin p \Delta \tau, \\
& \int_{p-k}^{p+k} d p^{\prime} \cos p^{\prime} \Delta \tau=\frac{1}{\Delta \tau}(\sin (p+k) \Delta \tau-\sin (p-k) \Delta \tau)=\frac{2}{\Delta \tau} \sin k \Delta \tau \cos p \Delta \tau
\end{aligned}
$$

Next we calculate the contributions

$$
\int_{M_{\mathrm{cm}}}^{\Lambda a\left(\tau_{2}\right)} d p p F_{i}\left(p, \tau_{1}, \tau_{2}\right) \int_{p-k}^{p+k} d p^{\prime} p^{\prime} G_{j}^{R}\left(p^{\prime}, \tau_{1}, \tau_{2}\right),
$$

for $i$ and $j$ from 1 to 3 :

\#1: $F_{1}\left(p, \tau_{1}, \tau_{2}\right) G_{1}^{R}\left(p^{\prime}, \tau_{1}, \tau_{2}\right)$

$$
\begin{aligned}
\int_{M_{\mathrm{cm}}}^{\Lambda a\left(\tau_{2}\right)} d p \frac{\cos p \Delta \tau}{p^{2}} \int_{p-k}^{p+k} d p^{\prime} \frac{\sin p^{\prime} \Delta \tau}{p^{\prime 2}}= \\
\quad\left[-\frac{\cos p \Delta \tau}{p}\left(-\frac{\sin (p+k) \Delta \tau}{p+k}+\frac{\sin (p-k) \Delta \tau}{p-k}+\right.\right. \\
\quad \int_{M_{\mathrm{cm}}}^{\Lambda a\left(\tau_{2}\right)} d p\left\{-\Delta \tau \frac{\sin p \Delta \tau}{p}\left(-\frac{\sin (p+k) \Delta \tau}{p+k}+\frac{\sin (p-k) \Delta \tau}{p-k}+\right.\right. \\
\Delta \tau(\operatorname{Ci}((p+k) \Delta \tau)-\operatorname{Ci}((p-k) \Delta \tau)))+ \\
\left.\frac{\cos p \Delta \tau}{p}\left(\frac{\sin (p+k) \Delta \tau}{(p+k)^{2}}-\frac{\sin (p-k) \Delta \tau}{(p-k)^{2}}\right)\right\}
\end{aligned}
$$


$\# 2: F_{1}\left(p, \tau_{1}, \tau_{2}\right) G_{2}^{R}\left(p^{\prime}, \tau_{1}, \tau_{2}\right)$

$$
\begin{aligned}
&-\Delta \tau \int_{M_{\mathrm{cm}}}^{\Lambda a\left(\tau_{2}\right)} d p \frac{\cos p \Delta \tau}{p^{2}} \int_{p-k}^{p+k} d p^{\prime} \frac{\cos p^{\prime} \Delta \tau}{p^{\prime}}= \\
& \Delta \tau\left[\frac{\cos p \Delta \tau}{p}(\operatorname{Ci}((p+k) \Delta \tau)-\operatorname{Ci}((p-k) \Delta \tau))\right]_{M_{\mathrm{cm}}}^{\Lambda a\left(\tau_{2}\right)}+ \\
& \Delta \tau \int_{M_{\mathrm{cm}}}^{\Lambda a\left(\tau_{2}\right)} d p\left\{\Delta \tau \frac{\sin p \Delta \tau}{p}(\operatorname{Ci}((p+k) \Delta \tau)-\operatorname{Ci}((p-k) \Delta \tau))+\right. \\
&\left.-\frac{\cos p \Delta \tau}{p}\left(\frac{\cos (p+k) \Delta \tau}{p+k}-\frac{\cos (p-k) \Delta \tau}{p-k}\right)\right\}
\end{aligned}
$$

$\# 3: F_{1}\left(p, \tau_{1}, \tau_{2}\right) G_{3}^{R}\left(p^{\prime}, \tau_{1}, \tau_{2}\right)$

$$
\begin{aligned}
& \tau_{1} \tau_{2} \int_{M_{\mathrm{cm}}}^{\Lambda a\left(\tau_{2}\right)} d p \frac{\cos p \Delta \tau}{p^{2}} \int_{p-k}^{p+k} d p^{\prime} \sin p^{\prime} \Delta \tau= \\
& \frac{\tau_{1} \tau_{2} \sin k \Delta \tau}{\Delta \tau} \int_{M_{\mathrm{cm}}}^{\Lambda a\left(\tau_{2}\right)} d p \frac{\sin 2 p \Delta \tau}{p^{2}}= \\
& \frac{\tau_{1} \tau_{2} \sin k \Delta \tau}{\Delta \tau}\left[-\frac{\sin 2 p \Delta \tau}{p}+2 \Delta \tau \operatorname{Ci}(2 p \Delta \tau)\right]_{M_{\mathrm{cm}}}^{\Lambda a\left(\tau_{2}\right)}
\end{aligned}
$$

\#4: $F_{2}\left(p, \tau_{1}, \tau_{2}\right) G_{1}^{R}\left(p^{\prime}, \tau_{1}, \tau_{2}\right)$

$$
\begin{aligned}
\Delta \tau \int_{M_{\mathrm{cm}}}^{\Lambda a\left(\tau_{2}\right)} d p \frac{\sin p \Delta \tau}{p} \int_{p-k}^{p+k} d p^{\prime} \frac{\sin p^{\prime} \Delta \tau}{p^{\prime 2}}= \\
\int_{M_{\mathrm{cm}}}^{\Lambda a\left(\tau_{2}\right)} d p \Delta \tau \frac{\sin p \Delta \tau}{p}\left(-\frac{\sin (p+k) \Delta \tau}{p+k}+\frac{\sin (p-k) \Delta \tau}{p-k}+\right. \\
\Delta \tau(\operatorname{Ci}((p+k) \Delta \tau)-\operatorname{Ci}((p-k) \Delta \tau)))
\end{aligned}
$$

\#5: $F_{2}\left(p, \tau_{1}, \tau_{2}\right) G_{2}^{R}\left(p^{\prime}, \tau_{1}, \tau_{2}\right)$

$$
\begin{aligned}
&-\Delta \tau^{2} \int_{M_{\mathrm{cm}}}^{\Lambda a\left(\tau_{2}\right)} d p \frac{\sin p \Delta \tau}{p} \int_{p-k}^{p+k} d p^{\prime} \frac{\cos p^{\prime} \Delta \tau}{p^{\prime}}= \\
&-\Delta \tau^{2} \int_{M_{\mathrm{cm}}}^{\Lambda a\left(\tau_{2}\right)} d p \frac{\sin p \Delta \tau}{p}(\operatorname{Ci}((p+k) \Delta \tau)-\operatorname{Ci}((p-k) \Delta \tau))
\end{aligned}
$$

\#6: $F_{2}\left(p, \tau_{1}, \tau_{2}\right) G_{3}^{R}\left(p^{\prime}, \tau_{1}, \tau_{2}\right)$

$$
\begin{gathered}
\Delta \tau \tau_{1} \tau_{2} \int_{M_{\mathrm{cm}}}^{\Lambda a\left(\tau_{2}\right)} d p \frac{\sin p \Delta \tau}{p} \int_{p-k}^{p+k} d p^{\prime} \sin p^{\prime} \Delta \tau= \\
\tau_{1} \tau_{2} \sin k \Delta \tau \int_{M_{\mathrm{cm}}}^{\Lambda a\left(\tau_{2}\right)} d p \frac{1-\cos 2 p \Delta \tau}{p}= \\
\tau_{1} \tau_{2} \sin k \Delta \tau[\ln p-\operatorname{Ci}(2 p \Delta \tau)]_{M_{\mathrm{cm}}}^{\Lambda a\left(\tau_{2}\right)}
\end{gathered}
$$


$\# 7: F_{3}\left(p, \tau_{1}, \tau_{2}\right) G_{1}^{R}\left(p^{\prime}, \tau_{1}, \tau_{2}\right)$

$$
\begin{gathered}
\tau_{1} \tau_{2} \int_{M_{\mathrm{cm}}}^{\Lambda a\left(\tau_{2}\right)} d p \cos p \Delta \tau \int_{p-k}^{p+k} d p^{\prime} \frac{\sin p^{\prime} \Delta \tau}{p^{\prime 2}}= \\
\tau_{1} \tau_{2} \int_{M_{\mathrm{cm}}}^{\Lambda a\left(\tau_{2}\right)} d p \cos p \Delta \tau\left(-\frac{\sin (p+k) \Delta \tau}{p+k}+\frac{\sin (p-k) \Delta \tau}{p-k}+\right. \\
\Delta \tau(\operatorname{Ci}((p+k) \Delta \tau)-\operatorname{Ci}((p-k) \Delta \tau)))= \\
-\frac{\tau_{1} \tau_{2}}{2}\left[\sin k \Delta \tau\left(\ln \left(p^{2}-k^{2}\right)-\operatorname{Ci}(2(p+k) \Delta \tau)-\operatorname{Ci}(2(p-k) \Delta \tau)\right)+\right. \\
\cos k \Delta \tau(\operatorname{Si}(2(p+k) \Delta \tau)-\operatorname{Si}(2(p-k) \Delta \tau))]_{M_{\mathrm{cm}}}^{\Lambda a\left(\tau_{2}\right)}+ \\
\tau_{1} \tau_{2} \Delta \tau \int_{M_{\mathrm{cm}}}^{\Lambda a\left(\tau_{2}\right)} d p \cos p \Delta \tau(\operatorname{Ci}((p+k) \Delta \tau)-\operatorname{Ci}((p-k) \Delta \tau)), \quad \text { D. }
\end{gathered}
$$

\#8: $F_{3}\left(p, \tau_{1}, \tau_{2}\right) G_{2}^{R}\left(p^{\prime}, \tau_{1}, \tau_{2}\right)$

$$
\begin{aligned}
-\tau_{1} \tau_{2} \Delta \tau & \int_{M_{\mathrm{cm}}}^{\Lambda a\left(\tau_{2}\right)} d p \cos p \Delta \tau \int_{p-k}^{p+k} d p^{\prime} \frac{\cos p^{\prime} \Delta \tau}{p^{\prime}}= \\
& -\tau_{1} \tau_{2} \Delta \tau \int_{M_{\mathrm{cm}}}^{\Lambda a\left(\tau_{2}\right)} d p \cos p \Delta \tau(\operatorname{Ci}((p+k) \Delta \tau)-\operatorname{Ci}((p-k) \Delta \tau))
\end{aligned}
$$

\#9: $F_{3}\left(p, \tau_{1}, \tau_{2}\right) G_{3}^{R}\left(p^{\prime}, \tau_{1}, \tau_{2}\right)$

$$
\tau_{1}^{2} \tau_{2}^{2} \int_{M_{\mathrm{cm}}}^{\Lambda a\left(\tau_{2}\right)} d p \cos p \Delta \tau \int_{p-k}^{p+k} d p^{\prime} \sin p^{\prime} \Delta \tau=\frac{\tau_{1}^{2} \tau_{2}^{2}}{\Delta \tau^{2}} \sin k \Delta \tau\left[\sin ^{2} p \Delta \tau\right]_{M_{\mathrm{cm}}}^{\Lambda a\left(\tau_{2}\right)}
$$

Together this becomes

$$
\begin{aligned}
& \begin{array}{l}
{\left[\frac{\cos p \Delta \tau}{p}\left(\frac{\sin (p+k) \Delta \tau}{p+k}-\frac{\sin (p-k) \Delta \tau}{p-k}\right)+\right.} \\
\frac{\tau_{1} \tau_{2} \sin k \Delta \tau}{2}(2 \operatorname{Ci}(2 p \Delta \tau)+\operatorname{Ci}(2(p+k) \Delta \tau)+\operatorname{Ci}(2(p-k) \Delta \tau)+ \\
\left.\quad \ln \frac{p^{2}}{p^{2}-k^{2}}-2 \frac{\sin 2 p \Delta \tau}{p \Delta \tau}\right)+ \\
\left.-\frac{\tau_{1} \tau_{2}}{2} \cos k \Delta \tau(\operatorname{Si}(2(p+k) \Delta \tau)-\operatorname{Si}(2(p-k) \Delta \tau))+\frac{\tau_{1}^{2} \tau_{2}^{2}}{\Delta \tau^{2}} \sin k \Delta \tau \sin ^{2} p \Delta \tau\right]_{M_{\mathrm{cm}}}^{\Lambda a\left(\tau_{2}\right)}+ \\
\int_{M_{\mathrm{cm}}}^{\Lambda a\left(\tau_{2}\right)} d p \frac{\cos p \Delta \tau}{p}\left\{\frac{\sin (p+k) \Delta \tau}{(p+k)^{2}}-\frac{\sin (p-k) \Delta \tau}{(p-k)^{2}}+\right. \\
\left.-\Delta \tau\left(\frac{\cos (p+k) \Delta \tau}{p+k}-\frac{\cos (p-k) \Delta \tau}{p-k}\right)\right\} .
\end{array}
\end{aligned}
$$

For the upper limit the boundary term vanishes as $1 / \Lambda^{2}$, except the last term which we will discuss below. The lower limit of the boundary term gives (where we use that $\left|M_{\mathrm{cm}} \tau_{i}\right| \ll 1$ )

$$
\frac{2}{3} k \Delta \tau^{3}-2 k \tau_{1} \tau_{2} \Delta \tau\left(-2+\gamma+\ln 2 M_{\mathrm{cm}} \Delta \tau\right)+\mathcal{O}\left(\tau_{i}^{4}\right)
$$


Using Mathematica, the integral in (D.31) becomes for $\left|M_{\mathrm{cm}} \tau_{i}\right| \ll 1$

$$
\frac{k \Delta \tau^{3}}{9}\left(8-6 \gamma-6 \ln 2 M_{\mathrm{cm}} \Delta \tau\right)+\mathcal{O}\left(\tau_{i}^{4}\right) .
$$

Together equation (D.31) becomes

$$
\frac{2 k}{3}\left(\tau_{1}^{3}-\tau_{2}^{3}\right)\left(\frac{7}{3}-\gamma-\ln 2 M_{\mathrm{cm}}\left(\tau_{1}-\tau_{2}\right)\right)-\frac{2}{3} k \tau_{1} \tau_{2}\left(\tau_{1}-\tau_{2}\right)+\frac{\tau_{1}^{2} \tau_{2}^{2}}{\Delta \tau^{2}} \sin k \Delta \tau \sin ^{2} \Lambda a\left(\tau_{2}\right) \Delta \tau+\mathcal{O}\left(\tau_{i}^{4}\right) .
$$

The term that contains the $\sin ^{2} \Lambda$ is logarithmically divergent for $\Lambda \rightarrow \infty$. This can be seen as follows. Consider the integral

$$
\int_{-\infty}^{\infty} d \Delta \tau \theta(\Delta \tau) f(\Delta \tau) \frac{\sin ^{2} \Lambda a\left(\tau_{2}\right) \Delta \tau}{\Delta \tau}=\frac{1}{2} \int_{0}^{\infty} d \Delta \tau f(\Delta \tau) \frac{1-\cos \left(\frac{-2 \Lambda}{H} \frac{\Delta \tau}{\tau_{1}-\Delta \tau}\right)}{\Delta \tau},
$$

where $f(\Delta \tau)$ is a test function. The integral can be split up into two integrals

$$
\int_{0}^{\infty}=\lim _{\varepsilon \rightarrow 0} \int_{\varepsilon}^{\eta}+\int_{\eta}^{\infty}
$$

where $\eta$ is used as a regulator time, which we take to zero in the end, after taking the limit $\Lambda \rightarrow \infty$. In the first integral we can approximate

$$
\frac{\Delta \tau}{\tau_{1}-\Delta \tau} \approx \frac{\Delta \tau}{\tau_{1}}, \quad f(\Delta \tau) \approx f(0)
$$

so that it becomes

$$
\begin{array}{r}
\lim _{\varepsilon \rightarrow 0} \int_{\varepsilon}^{\eta} d \Delta \tau f(0) \frac{1-\cos \left(\frac{-2 \Lambda}{H} \frac{\Delta \tau}{\tau_{1}}\right)}{\Delta \tau}=\lim _{\varepsilon \rightarrow 0} f(0)\left(\ln \frac{\eta}{\varepsilon}-\operatorname{Ci}\left(\frac{-2 \Lambda \eta}{H \tau_{1}}\right)+\operatorname{Ci}\left(\frac{-2 \Lambda \varepsilon}{H \tau_{1}}\right)\right)= \\
f(0)\left(\gamma+\ln \frac{-2 \Lambda \eta}{H \tau_{1}}\right)
\end{array}
$$

where we have taken $\mathrm{Ci}\left(-2 \Lambda \eta / H \tau_{1}\right) \rightarrow 0$, and $\mathrm{Ci}\left(-2 \Lambda \varepsilon / H \tau_{1}\right) \rightarrow \gamma+\ln \left(-2 \Lambda \varepsilon / H \tau_{1}\right)$. The remaining integral is

$$
\lim _{\Lambda \rightarrow \infty} \int_{\eta}^{\infty} d \Delta \tau f(\Delta \tau) \frac{1-\cos \left(\frac{-2 \Lambda}{H} \frac{\Delta \tau}{\tau_{1}-\Delta \tau}\right)}{\Delta \tau}=\int_{\eta}^{\infty} d \Delta \tau \frac{f(\Delta \tau)}{\Delta \tau}
$$

where the term with the cosine vanishes, provided that the test function $f(\Delta \tau)$ vanishes sufficiently fast as $\Delta \tau \rightarrow \infty$. Together we obtain for $\Lambda \rightarrow \infty$

$$
\begin{aligned}
& \int_{-\infty}^{\infty} d \Delta \tau \theta(\Delta \tau) f(\Delta \tau) \frac{\sin ^{2} \Lambda a\left(\tau_{2}\right) \Delta \tau}{\Delta \tau}= \\
& \int_{-\infty}^{\infty} d \Delta \tau f(\Delta \tau) \frac{1}{2}\left[\frac{\theta(-\eta+\Delta \tau)}{\Delta \tau}+\delta(\Delta \tau)\left(\gamma+\ln \frac{-2 \Lambda \eta}{H \tau_{1}}\right)\right]
\end{aligned}
$$

which is in the language of distributions

$$
\theta(\Delta \tau) \frac{\sin ^{2} \Lambda a\left(\tau_{2}\right) \Delta \tau}{\Delta \tau}=\frac{1}{2}\left[\frac{\theta(-\eta+\Delta \tau)}{\Delta \tau}+\delta(\Delta \tau)\left(\gamma+\ln \frac{-2 \Lambda \eta}{H \tau_{1}}\right)\right]
$$


Using this result in equation (D.34), gathering the right prefactors and adding the contribution from the counterterm (D.4), we obtain for the large momentum contribution

$$
\begin{aligned}
\frac{i \lambda^{2} \theta\left(\tau_{1}-\tau_{2}\right)}{2(2 \pi)^{2} H^{4}\left(\tau_{1} \tau_{2}\right)^{4}}\left(\frac{2}{3}\left(\tau_{1}^{3}-\tau_{2}^{3}\right)\right. & \left(\frac{7}{3}-\gamma-\ln 2 M_{\mathrm{cm}} \Delta \tau\right)-\frac{2}{3} \tau_{1} \tau_{2}\left(\tau_{1}-\tau_{2}\right)+ \\
& \left.\frac{\left(\tau_{1} \tau_{2}\right)^{2}}{2}\left[\frac{\theta(-\eta+\Delta \tau)}{\Delta \tau}+\delta(\Delta \tau)\left(\gamma+\ln \frac{-2 \mu \eta}{H \tau_{1}}\right)\right]\right)
\end{aligned}
$$

\section{D.1.3 Attaching the external lines}

Adding the small and large momenta contributions, we obtain for the amputated diagrams A and D:

$$
\begin{aligned}
\mathrm{A}_{\mathrm{amp}}\left(k, \tau_{1}, \tau_{2}\right) & +\mathrm{D}_{\mathrm{amp}}\left(k, \tau_{1}, \tau_{2}\right)=\frac{i \lambda^{2} \theta\left(\tau_{1}-\tau_{2}\right)}{6(2 \pi)^{2} H^{4}\left(\tau_{1} \tau_{2}\right)^{4}}\left(\left(\tau_{1}^{3}-\tau_{2}^{3}\right)\left(\frac{1}{\epsilon}+\frac{14}{3}-2 \gamma\right)+\right. \\
- & 2 \tau_{1} \tau_{2}\left(\tau_{1}-\tau_{2}\right)+2 \tau_{1}^{3} \ln \left|\frac{\tau_{2}}{2\left(\tau_{1}-\tau_{2}\right)}\right|-2 \tau_{2}^{3} \ln \left|\frac{\tau_{1}}{2\left(\tau_{1}-\tau_{2}\right)}\right|+ \\
\frac{3}{2}\left(\tau_{1} \tau_{2}\right)^{2} & {\left.\left[\frac{\theta\left(-\eta+\tau_{1}-\tau_{2}\right)}{\tau_{1}-\tau_{2}}+\delta\left(\tau_{1}-\tau_{2}\right)\left(\gamma+\ln \frac{-2 \mu \eta}{H \tau_{1}}\right)\right]+\mathcal{O}\left(\tau_{i}^{4}\right)+\mathcal{O}(\epsilon)\right), }
\end{aligned}
$$

where the dependence on $M_{\mathrm{cm}}$ has dropped out. The full correlation function is obtained by

$$
-i \int_{\tau_{H}}^{\tau} d \tau_{1} \int_{\tau_{H}}^{\tau} d \tau_{2} G^{R}\left(k, \tau, \tau_{1}\right) F\left(k, \tau, \tau_{2}\right)\left(\mathrm{A}_{\mathrm{amp}}\left(k, \tau_{1}, \tau_{2}\right)+\mathrm{D}_{\mathrm{amp}}\left(k, \tau_{1}, \tau_{2}\right)\right) .
$$

Because the external momentum $k$ is small, i.e. $\left|k \tau_{i}\right| \ll 1$, we can use the expanded versions of the two point functions (2.37), (2.38) (or the ones of (D.9), (D.10), but this gives only corrections of order $\mathcal{O}(\epsilon))$. Using the integrals

$$
\begin{aligned}
& \int_{\tau_{H}}^{\tau} d \tau_{1} \int_{\tau_{H}}^{\tau_{1}} d \tau_{2} \frac{\left(\tau^{3}-\tau_{1}^{3}\right)\left(\tau_{1}^{3}-\tau_{2}^{3}\right)}{\left(\tau_{1} \tau_{2}\right)^{4}}=\frac{1}{3}\left(1+2 \ln \frac{\tau}{\tau_{H}}+\frac{3}{2} \ln ^{2} \frac{\tau}{\tau_{H}}\right)+\mathcal{O}\left(\frac{\tau}{\tau_{H}}\right), \\
& \int_{\tau_{H}}^{\tau} d \tau_{1} \int_{\tau_{H}}^{\tau_{1}} d \tau_{2} \frac{\left(\tau^{3}-\tau_{1}^{3}\right)\left(\tau_{1}-\tau_{2}\right)}{\left(\tau_{1} \tau_{2}\right)^{3}}=-\frac{1}{12}\left(11+6 \ln \frac{\tau}{\tau_{H}}\right)+\mathcal{O}\left(\frac{\tau}{\tau_{H}}\right), \\
& \int_{\tau_{H}}^{\tau} d \tau_{1} \int_{\tau_{H}}^{\tau_{1}} d \tau_{2} \frac{\left(\tau^{3}-\tau_{1}^{3}\right)}{\left(\tau_{1} \tau_{2}\right)^{4}}\left(\tau_{1}^{3} \ln \left|\frac{\tau_{2}}{2\left(\tau_{1}-\tau_{2}\right)}\right|-\tau_{2}^{3} \ln \left|\frac{\tau_{1}}{2\left(\tau_{1}-\tau_{2}\right)}\right|\right)=\frac{1}{18}\left(\frac{97}{6}-18 \zeta(3)+\right. \\
& \left.\quad-2 \pi^{2}-6 \ln 2+\left(13-3 \pi^{2}-12 \ln 2\right) \ln \frac{\tau}{\tau_{H}}+(3-9 \ln 2) \ln ^{2} \frac{\tau}{\tau_{H}}+3 \ln ^{3} \frac{\tau}{\tau_{H}}\right)+\mathcal{O}\left(\frac{\tau}{\tau_{H}}\right),
\end{aligned}
$$

$$
\begin{gathered}
\int_{\tau_{H}}^{\tau} d \tau_{1} \int_{\tau_{H}}^{\tau_{1}} d \tau_{2} \frac{\left(\tau^{3}-\tau_{1}^{3}\right)}{\left(\tau_{1} \tau_{2}\right)^{2}}\left[\frac{\theta\left(-\eta+\tau_{1}-\tau_{2}\right)}{\tau_{1}-\tau_{2}}+\delta\left(\tau_{1}-\tau_{2}\right)\left(\gamma+\ln \frac{-2 \mu \eta}{H \tau_{1}}\right)\right]= \\
\frac{1}{6}\left(8-2 \gamma-\pi^{2}-2 \ln \frac{2 \mu}{H}+6\left(1-\gamma-\ln \frac{2 \mu}{H}\right) \ln \frac{\tau}{\tau_{H}}\right)+\mathcal{O}\left(\frac{\tau}{\tau_{H}}\right)
\end{gathered}
$$


(recall that $\eta$ is sent to zero), this becomes

$$
\begin{aligned}
\frac{\lambda^{2}}{36(2 \pi)^{2} k^{3}}\{ & \frac{1}{3 \epsilon}+\frac{194}{27}-\frac{7}{6} \gamma-\frac{17}{36} \pi^{2}-\frac{2}{3} \ln 2-2 \zeta(3)-\frac{1}{2} \ln \frac{2 \mu}{H}+ \\
& \left(\frac{2}{3 \epsilon}+\frac{127}{18}-\frac{17}{6} \gamma-\frac{1}{3} \pi^{2}-\frac{4}{3} \ln 2-\frac{3}{2} \ln \frac{2 \mu}{H}\right) \ln \frac{\tau}{\tau_{H}}+ \\
& \left.\left(\frac{1}{2 \epsilon}+\frac{8}{3}-\gamma-\ln 2\right) \ln ^{2} \frac{\tau}{\tau_{H}}+\frac{1}{3} \ln ^{3} \frac{\tau}{\tau_{H}}+\mathcal{O}\left(\frac{\tau}{\tau_{H}}\right)+\mathcal{O}(\epsilon)\right\} .
\end{aligned}
$$

There is an equal contribution from the diagram with $\tau_{1}$ and $\tau_{2}$ interchanged. Note that there is no dependence on $\ln k / \mu$ for $|k \tau| \ll 1$.

\section{D.2 Diagrams B and C}

The amputated versions of the diagrams with two external $G^{R}$ propagators are
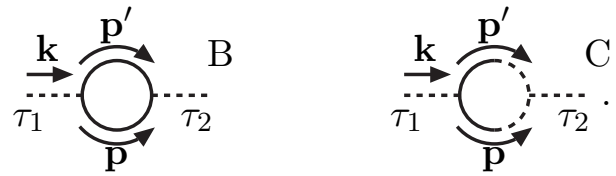

They translate to

$$
\begin{aligned}
\mathrm{B}_{\mathrm{amp}}\left(k, \tau_{1}, \tau_{2}\right) & =\frac{(-i \lambda)^{2}}{2 H^{8}\left(\tau_{1} \tau_{2}\right)^{4}} \int \frac{d^{3} p d^{3} p^{\prime}}{(2 \pi)^{3}} \delta^{3}\left(\mathbf{k}-\mathbf{p}-\mathbf{p}^{\prime}\right) F\left(p^{\prime}, \tau_{1}, \tau_{2}\right) F\left(p, \tau_{1}, \tau_{2}\right) \\
& =\frac{-\lambda^{2}}{2(2 \pi)^{2} k H^{8}\left(\tau_{1} \tau_{2}\right)^{4}} \int_{0}^{\infty} d p p \int_{|p-k|}^{p+k} d p^{\prime} p^{\prime} F\left(p^{\prime}, \tau_{1}, \tau_{2}\right) F\left(p, \tau_{1}, \tau_{2}\right), \\
\mathrm{C}_{\mathrm{amp}}\left(k, \tau_{1}, \tau_{2}\right) & =\frac{(-i)^{2}(-i \lambda)^{2}}{8 H^{8}\left(\tau_{1} \tau_{2}\right)^{4}} \int \frac{d^{3} p d^{3} p^{\prime}}{(2 \pi)^{3}} \delta^{3}\left(\mathbf{k}-\mathbf{p}-\mathbf{p}^{\prime}\right) G^{R}\left(p^{\prime}, \tau_{1}, \tau_{2}\right) G^{R}\left(p, \tau_{1}, \tau_{2}\right) \\
& =\frac{\lambda^{2}}{8(2 \pi)^{2} k H^{8}\left(\tau_{1} \tau_{2}\right)^{4}} \int_{0}^{\infty} d p p \int_{|p-k|}^{p+k} d p^{\prime} p^{\prime} G^{R}\left(p^{\prime}, \tau_{1}, \tau_{2}\right) G^{R}\left(p, \tau_{1}, \tau_{2}\right),
\end{aligned}
$$

where both diagrams have a factor $1 / 2$ for symmetry. Diagram $\mathrm{C}$ has an additional factor $1 / 4$ from the vertex with three dashed lines (2.29). We split the $p$ integral again in a small momentum part and a large momentum part.

\section{D.2.1 Amputated diagrams for small internal momenta}

For small internal momenta we use the expanded propagators (D.9) and (D.10).

Diagram B. The integral is

$$
\begin{aligned}
& \frac{-\lambda^{2}}{2(2 \pi)^{2} k H^{8}\left(\tau_{1} \tau_{2}\right)^{4}} \frac{H^{4}\left(\tau_{1} \tau_{2}\right)^{2 \epsilon}}{4} \int_{0}^{M_{\mathrm{cm}}} d p \int_{|p-k|}^{p+k} d p^{\prime} \frac{\left(p p^{\prime}\right)^{2 \epsilon}}{\left(p p^{\prime}\right)^{2}}= \\
& \frac{-\lambda^{2}\left(\tau_{1} \tau_{2}\right)^{2 \epsilon}}{8(2 \pi)^{2} k H^{4}\left(\tau_{1} \tau_{2}\right)^{4}(2 \epsilon-1)}\left(\int_{0}^{k} d p p^{-2+2 \epsilon}\left((p+k)^{-1+2 \epsilon}-(k-p)^{-1+2 \epsilon}\right)+\right. \\
& \left.\int_{k}^{M_{\mathrm{cm}}} d p p^{-2+2 \epsilon}\left((p+k)^{-1+2 \epsilon}-(p-k)^{-1+2 \epsilon}\right)\right) .
\end{aligned}
$$


The integral on the middle line of (D.53) is finite, but the individual parts are infrared divergent. Therefore we calculate the individual parts for $\epsilon>1 / 2$, and in the end use analytic continuation to $\epsilon \ll 1$. The integrals are (using $p=k x$ )

$$
\begin{aligned}
\int_{0}^{1} d x x^{-2+2 \epsilon}(1+x)^{-1+2 \epsilon} & =\sum_{n=0}^{\infty} \frac{(-1)^{n}}{n !} \frac{\Gamma(1+n-2 \epsilon)}{\Gamma(1-2 \epsilon)} \int_{0}^{1} d x x^{-2+n+2 \epsilon} \\
& =-\frac{1}{2 \epsilon}+\ln 2+\mathcal{O}(\epsilon) \\
\int_{0}^{1} d x x^{-2+2 \epsilon}(1-x)^{-1+2 \epsilon} & =B(-1+2 \epsilon, 2 \epsilon)=\frac{1}{\epsilon}-2+\mathcal{O}(\epsilon), \\
\int_{1}^{M_{\mathrm{cm}} / k} d x x^{-2+2 \epsilon}(1+x)^{-1+2 \epsilon} & =\int_{k / M_{\mathrm{cm}}}^{1} d y \frac{y^{1-4 \epsilon}}{(1+y)^{1-2 \epsilon}} \\
& =1-\frac{k}{M_{\mathrm{cm}}}-\ln 2+\ln \left(1+\frac{k}{M_{\mathrm{cm}}}\right)+\mathcal{O}(\epsilon), \\
\int_{1}^{M_{\mathrm{cm} / k}} d x x^{-2+2 \epsilon}(x-1)^{-1+2 \epsilon} & =\int_{k / M_{\mathrm{cm}}}^{1} d y \frac{y^{1-4 \epsilon}}{(1-y)^{1-2 \epsilon}}=\int_{0}^{1-k / M_{\mathrm{cm}}} d z z^{-1+2 \epsilon}(1-z)+\mathcal{O}(\epsilon) \\
& =\frac{1}{2 \epsilon}-1+\frac{k}{M_{\mathrm{cm}}}+\ln \left(1-\frac{k}{M_{\mathrm{cm}}}\right)+\mathcal{O}(\epsilon),
\end{aligned}
$$

where we have used analytic continuation in the first two integrals and $y=1 / x$ and $z=1-y$ in the latter two. The right hand side of equation (D.53) becomes

$$
\begin{aligned}
\frac{-\lambda^{2}\left(k^{2} \tau_{1} \tau_{2}\right)^{2 \epsilon}}{8(2 \pi)^{2} k^{3} H^{4}\left(\tau_{1} \tau_{2}\right)^{4}(2 \epsilon-1)}\left(\frac{-2}{\epsilon}+4-2 \frac{k}{M_{\mathrm{cm}}}+\ln \frac{1+k / M_{\mathrm{cm}}}{1-k / M_{\mathrm{cm}}}+\mathcal{O}(\epsilon)\right)= \\
\frac{-\lambda^{2}}{4(2 \pi)^{2} k^{3} H^{4}\left(\tau_{1} \tau_{2}\right)^{4}}\left(\frac{1}{\epsilon}+\frac{k}{M_{\mathrm{cm}}}-\frac{1}{2} \ln \frac{M_{\mathrm{cm}}+k}{M_{\mathrm{cm}}-k}+2 \ln \left(k^{2} \tau_{1} \tau_{2}\right)+\mathcal{O}(\epsilon)\right) .
\end{aligned}
$$

Diagram C. From equations (D.52) and (D.10) we see directly that diagram C does not give late time contributions and also does not have an infrared divergence.

\section{D.2.2 Amputated diagrams for large internal momenta}

The contributions from large internal momenta can be calculated in a similar way as is used for diagram A in section D.1.2.

Diagram B. The sum of integrals

$$
\sum_{i=1}^{3} \sum_{j=1}^{3} \int_{M_{\mathrm{cm}}}^{\Lambda a\left(\tau_{\beta}\right)} d p p F_{i}\left(p, \tau_{1}, \tau_{2}\right) \int_{p-k}^{p+k} d p^{\prime} p^{\prime} F_{j}\left(p^{\prime}, \tau_{1}, \tau_{2}\right)
$$


(where $\tau_{\beta}=\tau_{1}, \tau_{2}$, depending on which time is earlier), is equal to

$$
\begin{aligned}
& {\left[\frac{\cos p \Delta \tau}{p}\left(\frac{\cos (p+k) \Delta \tau}{p+k}-\frac{\cos (p-k) \Delta \tau}{p-k}\right)+\right.} \\
& \left.\quad-\tau_{1} \tau_{2} \sin k \Delta \tau\left(\operatorname{Si}(2 p \Delta \tau)+2 \frac{\cos ^{2} p \Delta \tau}{p \Delta \tau}\right)+\frac{\left(\tau_{1} \tau_{2}\right)^{2}}{\Delta \tau} \sin k \Delta \tau\left(p+\frac{\sin 2 p \Delta \tau}{2 \Delta \tau}\right)\right]_{M_{\mathrm{cm}}}^{\Lambda a\left(\tau_{\beta}\right)}+ \\
& \int_{M_{\mathrm{cm}}}^{\Lambda a\left(\tau_{\beta}\right)} d p \frac{\cos p \Delta \tau}{p}\left\{\frac{\cos (p+k) \Delta \tau}{(p+k)^{2}}-\frac{\cos (p-k) \Delta \tau}{(p-k)^{2}}+\right. \\
& \left.\quad \Delta \tau\left(\frac{\sin (p+k) \Delta \tau}{p+k}-\frac{\sin (p-k) \Delta \tau}{p-k}\right)-p \tau_{1} \tau_{2}\left(\frac{\cos (p+k) \Delta \tau}{p+k}-\frac{\cos (p-k) \Delta \tau}{p-k}\right)\right\} .
\end{aligned}
$$

The only ultraviolet term comes from the last term of the boundary term and is, including the correct prefactor:

$$
\frac{-\lambda^{2} \sin k \Delta \tau}{8(2 \pi)^{2} k H^{4}\left(\tau_{1} \tau_{2}\right)^{2} \Delta \tau}\left[p+\frac{\sin 2 p \Delta \tau}{2 \Delta \tau}\right]_{M_{\mathrm{cm}}}^{\Lambda a\left(\tau_{\beta}\right)} .
$$

The only term that gives late time contributions is the first line in the integral. It is

$$
\frac{-\lambda^{2}}{4(2 \pi)^{2} k^{3} H^{4}\left(\tau_{1} \tau_{2}\right)^{4}}\left(-\frac{k}{M_{\mathrm{cm}}}+\frac{1}{2} \ln \frac{M_{\mathrm{cm}}+k}{M_{\mathrm{cm}}-k}+\mathcal{O}\left(\tau_{i}^{2}\right)\right) .
$$

Diagram C. The sum of integrals

$$
\sum_{i=1}^{3} \sum_{j=1}^{3} \int_{M_{\mathrm{cm}}}^{\Lambda a\left(\tau_{\beta}\right)} d p p G_{i}^{R}\left(p, \tau_{1}, \tau_{2}\right) \int_{p-k}^{p+k} d p^{\prime} p^{\prime} G_{j}^{R}\left(p^{\prime}, \tau_{1}, \tau_{2}\right)
$$

is equal to

$$
\begin{aligned}
& \quad \frac{\sin p \Delta \tau}{p}\left(\frac{\sin (p+k) \Delta \tau}{p+k}-\frac{\sin (p-k) \Delta \tau}{p-k}\right)+ \\
& \left.\quad+\tau_{1} \tau_{2} \sin k \Delta \tau\left(3 \operatorname{Si}(2 p \Delta \tau)-2 \frac{\sin ^{2} p \Delta \tau}{p \Delta \tau}\right)+\frac{\left(\tau_{1} \tau_{2}\right)^{2}}{\Delta \tau} \sin k \Delta \tau\left(p-\frac{\sin 2 p \Delta \tau}{2 \Delta \tau}\right)\right]_{M_{\mathrm{cm}}}^{\Lambda a\left(\tau_{\beta}\right)}+ \\
& \int_{M_{\mathrm{cm}}}^{\Lambda a\left(\tau_{\beta}\right)} d p \frac{\sin p \Delta \tau}{p}\left\{\frac{\sin (p+k) \Delta \tau}{(p+k)^{2}}-\frac{\sin (p-k) \Delta \tau}{(p-k)^{2}}+\right. \\
& \left.\quad-\Delta \tau\left(\frac{\cos (p+k) \Delta \tau}{p+k}-\frac{\cos (p-k) \Delta \tau}{p-k}\right)-p \tau_{1} \tau_{2}\left(\frac{\sin (p+k) \Delta \tau}{p+k}-\frac{\sin (p-k) \Delta \tau}{p-k}\right)\right\} .
\end{aligned}
$$

Only the last term of the boundary term is ultraviolet divergent:

$$
\frac{\lambda^{2} \theta\left(\tau_{1}-\tau_{2}\right) \sin k \Delta \tau}{8(2 \pi)^{2} k H^{4}\left(\tau_{1} \tau_{2}\right)^{2} \Delta \tau}\left[p-\frac{\sin 2 p \Delta \tau}{2 \Delta \tau}\right]_{M_{\mathrm{cm}}}^{\Lambda a\left(\tau_{\beta}\right)} .
$$

The diagram with the vertices exchanged gives the same result, except that $\theta\left(\tau_{1}-\tau_{2}\right)$ is replaced by $\theta\left(\tau_{2}-\tau_{1}\right)$. There are no further late time contributions. 
Ultraviolet divergences. The ultraviolet divergent terms of diagrams B (D.61), C (D.65), and $\mathrm{C}$ with the vertices exchanged, add up to

$$
\left[\frac{-\lambda^{2} \sin k \Delta \tau}{8(2 \pi)^{2} k H^{4}\left(\tau_{1} \tau_{2}\right)^{2} \Delta \tau} \frac{\sin 2 p \Delta \tau}{\Delta \tau}\right]_{M_{\mathrm{cm}}}^{\Lambda a\left(\tau_{\beta}\right)}
$$

which is finite and does not give late time contributions.

\section{D.2.3 Attaching the external lines}

Adding the small and large momenta contributions, we obtain for the amputated diagrams $\mathrm{B}$ and $\mathrm{C}$ :

$$
\mathrm{B}_{\mathrm{amp}}\left(k, \tau_{1}, \tau_{2}\right)+\mathrm{C}_{\mathrm{amp}}\left(k, \tau_{1}, \tau_{2}\right)=\frac{-\lambda^{2}}{4(2 \pi)^{2} k^{3} H^{4}\left(\tau_{1} \tau_{2}\right)^{4}}\left(\frac{1}{\epsilon}+2 \ln \left(k^{2} \tau_{1} \tau_{2}\right)+\mathcal{O}\left(\tau_{i}\right)+\mathcal{O}(\epsilon)\right),
$$

where the dependence on $M_{\mathrm{cm}}$ has dropped out. The full correlation function is obtained by

$$
-\int_{\tau_{H}}^{\tau} d \tau_{1} \int_{\tau_{H}}^{\tau} d \tau_{2} G^{R}\left(k, \tau, \tau_{1}\right) G^{R}\left(k, \tau, \tau_{2}\right)\left(\mathrm{B}_{\mathrm{amp}}\left(k, \tau_{1}, \tau_{2}\right)+\mathrm{C}_{\mathrm{amp}}\left(k, \tau_{1}, \tau_{2}\right)\right) .
$$

Because the external momentum $k$ is small, i.e. $\left|k \tau_{i}\right| \ll 1$, we can use the expanded version of the $G^{R}$ propagator (2.38) (or the one of (D.10), but this gives only corrections of order $\mathcal{O}(\epsilon))$. Using the integrals

$$
\begin{gathered}
\int_{\tau_{H}}^{\tau} d \tau_{1} \int_{\tau_{H}}^{\tau} d \tau_{2} \frac{\left(\tau^{3}-\tau_{1}^{3}\right)\left(\tau^{3}-\tau_{2}^{3}\right)}{\left(\tau_{1} \tau_{2}\right)^{4}}=\frac{1}{9}+\frac{2}{3} \ln \frac{\tau}{\tau_{H}}+\ln ^{2} \frac{\tau}{\tau_{H}}+\mathcal{O}\left(\frac{\tau}{\tau_{H}}\right), \\
\int_{\tau_{H}}^{\tau} d \tau_{1} \int_{\tau_{H}}^{\tau} d \tau_{2} \frac{\left(\tau^{3}-\tau_{1}^{3}\right)\left(\tau^{3}-\tau_{2}^{3}\right)}{\left(\tau_{1} \tau_{2}\right)^{4}} \ln \left(k^{2} \tau_{1} \tau_{2}\right)=\frac{1}{27}\left(2+6 \ln \left(-k \tau_{H}\right)+\right. \\
\left.12\left(1+3 \ln \left(-k \tau_{H}\right)\right) \ln \frac{\tau}{\tau_{H}}+27\left(1+2 \ln \left(-k \tau_{H}\right)\right) \ln ^{2} \frac{\tau}{\tau_{H}}+27 \ln ^{3} \frac{\tau}{\tau_{H}}\right)+\mathcal{O}\left(\frac{\tau}{\tau_{H}}\right),
\end{gathered}
$$

this becomes

$$
\begin{array}{r}
\frac{\lambda^{2}}{36(2 \pi)^{2} k^{3}}\left(\frac{1}{9 \epsilon}+\frac{4}{27}+\frac{4}{9} \ln \left(-k \tau_{H}\right)+\left(\frac{2}{3 \epsilon}+\frac{8}{9}+\frac{8}{3} \ln \left(-k \tau_{H}\right)\right) \ln \frac{\tau}{\tau_{H}}+\right. \\
\left.\left(\frac{1}{\epsilon}+2+4 \ln \left(-k \tau_{H}\right)\right) \ln ^{2} \frac{\tau}{\tau_{H}}+2 \ln ^{3} \frac{\tau}{\tau_{H}}+\mathcal{O}\left(\frac{\tau}{\tau_{H}}\right)+\mathcal{O}(\epsilon)\right) .
\end{array}
$$

\section{References}

[1] N. Bartolo, E. Komatsu, S. Matarrese, and A. Riotto, Non-Gaussianity from inflation: Theory and observations, Phys. Rept. 402 (2004) 103-266, astro-ph/0406398.

[2] J. M. Bardeen, Gauge invariant cosmological perturbations, Phys. Rev. D22 (1980) $1882-1905$.

[3] J. M. Maldacena, Non-Gaussian features of primordial fluctuations in single field inflationary models, JHEP 05 (2003) 013, astro-ph/0210603. 
[4] D. H. Lyth, K. A. Malik, and M. Sasaki, A general proof of the conservation of the curvature perturbation, JCAP 0505 (2005) 004, astro-ph/0411220.

[5] F. Bernardeau and J.-P. Uzan, Non-Gaussianity in multi-field inflation, Phys. Rev. D66 (2002) 103506, hep-ph/0207295.

[6] F. Bernardeau and J.-P. Uzan, Inflationary models inducing non-Gaussian metric fluctuations, Phys. Rev. D67 (2003) 121301, astro-ph/0209330].

[7] K. Enqvist and A. Vaihkonen, Non-Gaussian perturbations in hybrid inflation, JCAP 0409 (2004) 006, hep-ph/0405103.

[8] G. I. Rigopoulos, E. P. S. Shellard, and B. J. W. van Tent, A simple route to non-Gaussianity in inflation, Phys. Rev. D72 (2005) 083507, astro-ph/0410486.

[9] G. I. Rigopoulos, E. P. S. Shellard, and B. J. W. van Tent, Non-linear perturbations in multiple-field inflation, Phys. Rev. D73 (2006) 083521, [astro-ph/0504508].

[10] G. I. Rigopoulos, E. P. S. Shellard, and B. W. van Tent, Large non-Gaussianity in multiple-field inflation, Phys. Rev. D73 (2006) 083522, [astro-ph/0506704.

[11] G. I. Rigopoulos, E. P. S. Shellard, and B. J. W. van Tent, Quantitative bispectra from multifield inflation, astro-ph/0511041.

[12] D. H. Lyth and Y. Rodriguez, Non-Gaussianity from the second-order cosmological perturbation, Phys. Rev. D71 (2005) 123508, astro-ph/0502578].

[13] D. H. Lyth and Y. Rodriguez, The inflationary prediction for primordial non- Gaussianity, Phys. Rev. Lett. 95 (2005) 121302, astro-ph/0504045.

[14] I. Zaballa, Y. Rodriguez, and D. H. Lyth, Higher order contributions to the primordial nonGaussianity, JCAP 0606 (2006) 013, [estro-ph/0603534.

[15] F. Vernizzi and D. Wands, Non-Gaussianities in two-field inflation, JCAP 0605 (2006) 019, astro-ph/0603799.

[16] L. E. Allen, S. Gupta, and D. Wands, Non-Gaussian perturbations from multi-field inflation, JCAP 0601 (2006) 006, astro-ph/0509719.

[17] H.-C. Lee, M. Sasaki, E. D. Stewart, T. Tanaka, and S. Yokoyama, A new delta $N$ formalism for multi-component inflation, JCAP 0510 (2005) 004, astro-ph/0506262.

[18] S. A. Kim and A. R. Liddle, Nflation: Non-Gaussianity in the horizon-crossing approximation, Phys. Rev. D74 (2006) 063522, astro-ph/0608186.

[19] C. T. Byrnes, M. Sasaki, and D. Wands, The primordial trispectrum from inflation, Phys. Rev. D74 (2006) 123519, astro-ph/0611075.

[20] D. Seery and J. E. Lidsey, Non-Gaussianity from the inflationary trispectrum, JCAP 0701 (2007) 008, astro-ph/0611034.

[21] L. Alabidi, Non-Gaussianity for a two component hybrid model of inflation, JCAP $06 \mathbf{0 1 0}$ (2006) 015, astro-ph/0604611.

[22] D. Seery, J. E. Lidsey, and M. S. Sloth, The inflationary trispectrum, JCAP 0701 (2007) 027, astro-ph/0610210.

[23] T. Battefeld and R. Easther, Non-Gaussianities in multi-field inflation, JCAP 0703 (2007) 020, astro-ph/0610296. 
[24] D. Battefeld and T. Battefeld, Non-Gaussianities in N-flation, JCAP 0705 (2007) 012, hep-th/0703012.

[25] K.-Y. Choi, L. M. H. Hall, and C. van de Bruck, Spectral running and non-Gaussianity from slow-roll inflation in generalised two-field models, JCAP 0702 (2007) 029, astro-ph/0701247.

[26] S. Yokoyama, T. Suyama, and T. Tanaka, Primordial non-Gaussianity in multi-scalar slow-roll inflation, arXiv:0705.3178 [astro-ph].

[27] C. T. Byrnes, K. Koyama, M. Sasaki, and D. Wands, Diagrammatic approach to non-Gaussianity from inflation, arXiv:0705.4096 [hep-th].

[28] D. H. Lyth and D. Seery, Classicality of the primordial perturbations, astro-ph/0607647.

[29] E. Calzetta and B. L. Hu, Closed time path functional formalism in curved space-time: Application to cosmological back reaction problems, Phys. Rev. D35 (1987) 495.

[30] E. Calzetta and B. L. Hu, Nonequilibrium quantum fields: Closed time path effective action, Wigner function and Boltzmann equation, Phys. Rev. D37 (1988) 2878.

[31] S. Weinberg, Quantum contributions to cosmological correlations, Phys. Rev. D72 (2005) 043514, hep-th/0506236.

[32] S. Weinberg, Quantum contributions to cosmological correlations. II: Can these corrections become large?, Phys. Rev. D74 (2006) 023508, hep-th/0605244.

[33] D. Polarski and A. A. Starobinsky, Semiclassicality and decoherence of cosmological perturbations, Class. Quant. Grav. 13 (1996) 377-392, gr-qc/9504030.

[34] C. Kiefer, D. Polarski, and A. A. Starobinsky, Quantum-to-classical transition for fluctuations in the early universe, Int. J. Mod. Phys. D7 (1998) 455-462, gr-qc/9802003.

[35] C. Kiefer, I. Lohmar, D. Polarski, and A. A. Starobinsky, Pointer states for primordial fluctuations in inflationary cosmology, Class. Quant. Grav. 24 (2007) 1699-1718, astro-ph/0610700.

[36] P. Martineau, On the decoherence of primordial fluctuations during inflation, astro-ph/0601134.

[37] C. P. Burgess, R. Holman, and D. Hoover, On the decoherence of primordial fluctuations during inflation, astro-ph/0601646.

[38] T. Prokopec and G. I. Rigopoulos, Decoherence from isocurvature perturbations in inflation, astro-ph/0612067.

[39] N. C. Tsamis and R. P. Woodard, Strong infrared effects in quantum gravity, Ann. Phys. 238 (1995) $1-82$.

[40] N. C. Tsamis and R. P. Woodard, The quantum gravitational back-reaction on inflation, Annals Phys. 253 (1997) 1-54, hep-ph/9602316.

[41] V. K. Onemli and R. P. Woodard, Super-acceleration from massless, minimally coupled phi**4, Class. Quant. Grav. 19 (2002) 4607, gr-qc/0204065.

[42] T. Brunier, V. K. Onemli, and R. P. Woodard, Two loop scalar self-mass during inflation, Class. Quant. Grav. 22 (2005) 59-84, gr-qc/0408080. 
[43] V. K. Onemli and R. P. Woodard, Quantum effects can render $w<-1$ on cosmological scales, Phys. Rev. D70 (2004) 107301, gr-qc/0406098.

[44] E. O. Kahya and V. K. Onemli, Quantum stability of a $w<-1$ phase of cosmic acceleration, gr-qc/0612026.

[45] T. Prokopec, O. Tornkvist, and R. P. Woodard, One loop vacuum polarization in a locally de Sitter background, Ann. Phys. 303 (2003) 251-274, gr-qc/0205130.

[46] T. Prokopec and R. P. Woodard, Production of massless fermions during inflation, JHEP 10 (2003) 059, astro-ph/0309593.

[47] T. Prokopec, N. C. Tsamis, and R. P. Woodard, Two loop scalar bilinears for inflationary SQED, Class. Quant. Grav. 24 (2007) 201-230, gr-qc/0607094.

[48] D. Boyanovsky et al., Scalar field dynamics in Friedman Robertson Walker spacetimes, Phys. Rev. D56 (1997) 1939-1957, hep-ph/9703327].

[49] D. Boyanovsky and H. J. de Vega, Dynamical renormalization group approach to relaxation in quantum field theory, Ann. Phys. 307 (2003) 335-371, hep-ph/0302055.

[50] D. Boyanovsky and H. J. de Vega, Particle decay in inflationary cosmology, Phys. Rev. D70 (2004) 063508, astro-ph/0406287.

[51] D. Boyanovsky, H. J. de Vega, and N. G. Sanchez, Particle decay during inflation: Self-decay of inflaton quantum fluctuations during slow roll, Phys. Rev. D71 (2005) 023509, astro-ph/0409406.

[52] D. Boyanovsky, H. J. de Vega, and N. G. Sanchez, Quantum corrections to slow roll inflation and new scaling of superhorizon fluctuations, Nucl. Phys. B747 (2006) 25-54, astro-ph/0503669.

[53] M. S. Sloth, On the one loop corrections to inflation and the CMB anisotropies, Nucl. Phys. B748 (2006) 149-169, astro-ph/0604488.

[54] M. S. Sloth, On the one loop corrections to inflation. II: The consistency relation, Nucl. Phys. B775 (2007) 78-94, hep-th/0612138].

[55] K. Chaicherdsakul, Quantum cosmological correlations in an inflating universe: Can fermion and gauge fields loops give a scale free spectrum?, Phys. Rev. D75 (2007) 063522, hep-th/0611352.

[56] A. Bilandzic and T. Prokopec, Quantum radiative corrections to slow-roll inflation, arXiv:0704.1905 [astro-ph].

[57] G. Aarts and J. Smit, Classical approximation for time-dependent quantum field theory: Diagrammatic analysis for hot scalar fields, Nucl. Phys. B511 (1998) 451-478, hep-ph/9707342.

[58] P. R. Anderson, W. Eaker, S. Habib, C. Molina-Paris, and E. Mottola, Attractor states and infrared scaling in de sitter space, Phys. Rev. D62 (2000) 124019, gr-qc/0005102.

[59] P. R. Anderson, C. Molina-Paris, and E. Mottola, Short distance and initial state effects in inflation: Stress tensor and decoherence, Phys. Rev. D72 (2005) 043515, hep-th/0504134.

[60] M. Musso, A new diagrammatic representation for correlation functions in the in-in formalism, hep-th/0611258. 
[61] G. Aarts and J. Smit, Finiteness of hot classical scalar field theory and the plasmon damping rate, Phys. Lett. B393 (1997) 395-402, hep-ph/9610415.

[62] A. A. Starobinsky, Stochastic de Sitter (inflationary) stage in the early universe, in Field Theory, Quantum Gravity and Strings (Lecture Notes in Physics vol 246), editors H.J. De Vega and N. Sanchez, Berlin: Springer (1986) pp 107-126.

[63] A. A. Starobinsky and J. Yokoyama, Equilibrium state of a selfinteracting scalar field in the de sitter background, Phys. Rev. D50 (1994) 6357-6368, astro-ph/9407016.

[64] R. P. Woodard, A leading logarithm approximation for inflationary quantum field theory, Nucl. Phys. Proc. Suppl. 148 (2005) 108-119, astro-ph/0502556.

[65] N. C. Tsamis and R. P. Woodard, Stochastic quantum gravitational inflation, Nucl. Phys. B724 (2005) 295-328, gr-qc/0505115.

[66] S.-P. Miao and R. P. Woodard, Leading log solution for inflationary Yukawa, Phys. Rev. D74 (2006) 044019, gr-qc/0602110.

[67] L. R. W. Abramo and R. P. Woodard, One loop back reaction on power law inflation, Phys. Rev. D60 (1999) 044011, astro-ph/9811431.

[68] T. Prokopec, N. C. Tsamis, and R. P. Woodard, Stochastic inflationary scalar electrodynamics, arXiv:0707.0847 [gr-qc].

[69] N. D. Birrell and P. C. W. Davies, Quantum fields in curved space, Cambridge, UK: Univ. Pr., 340 p (1982).

[70] G. Aarts, B.-J. Nauta, and C. G. van Weert, Divergences in real-time classical field theories at non- zero temperature, Phys. Rev. D61 (2000) 105002, hep-ph/9911463.

[71] M. Abramowitz and I. A. Stegun (eds), Handbook of Mathematical Functions with Formulas, Graphs and Mathematical Tables, New York: Dover, ISBN 0-486-61272-4 (1965). 